\title{
Metal-Ligand Cooperation between Palladium and a Diphosphine Ligand With an Olefinic Backbone
}

\author{
Brittany J. Barrett and Vlad M. Iluc*
}

\begin{abstract}
The tridentate palladium $(0)$ compound $\quad[(c \mathrm{PCMe}=\mathrm{CMeP}) \mathrm{Pd}] \quad((\mathrm{Z})-2,3-\mathrm{bis}(2$-di-isopropylphosphinephenyl)-2-butene) was synthesized and its oxidative addition products with $\mathrm{CH}_{3} \mathrm{I}, \mathrm{C}_{6} \mathrm{H}_{5} \mathrm{I}, \mathrm{Ph}_{2} \mathrm{SiH}_{2}$, and $\mathrm{HCl}$ were characterized. Hemilability of the olefinic backbone is observed in all cases and, excluding $\mathrm{Ph}_{2} \mathrm{SiH}_{2}$, results in trans palladium(II) species. Oxidative addition of $\mathrm{Ph}_{2} \mathrm{SiH}_{2}$ results in a fluxional species due to the reversibility of the $\mathrm{Si}-\mathrm{H}$ bond activation. Reactivity with $\mathrm{HCl}$ led to the oxidative addition of the acid, resulting in the formation of a clorohydride complex, $[(c \mathrm{PCMe}=\mathrm{CMeP}) \mathrm{PdHCl}]$, followed by a irreversible insertion of the olefin into the $\mathrm{Pd}-\mathrm{H}$ bond resulting in [(PCMe-CHMeP)PdCl$]$. Additionally, dehydrohalogenation was achieved with $\left[(c \mathrm{PCMe}=\mathrm{CMeP}) \mathrm{PdCl}_{2}\right]$ resulting in $[(\mathrm{PCMe}-$ $\left.\mathrm{C}\left(=\mathrm{CH}_{2}\right) \mathrm{P}\right) \mathrm{PdCl}$. All metal complexes were characterized by multi-nuclei NMR spectroscopy, X-ray crystallography, and elemental analysis.
\end{abstract}

Keywords: hemilabile; trans chelating ligand; diphospine; palladium 


\section{Introduction}

The use of metal-ligand cooperation in organometallic chemistry has become an area of great interest. Utilizing ligands capable of participating in reactions has led to some useful bond activation processes. ${ }^{1-5}$ In particular, ligand frameworks capable of accepting/donating hydrogen atoms have aided in a variety of catalytic cycles. ${ }^{6-8}$ Milstein's pioneering work using pyridine based PNP and PNN pincer ligands invokes an aromatization/dearomatization process upon hydrogen atom acceptance and donation and has contributed to vast improvements in catalyzing reactions, such as the dehydrogenation of alcohols to esters and acylation of amines by alcohols. $^{7-9}$ Examples such as these have inspired the design of various ligands with H-atom acceptor and donor capabilities. For example, a $p$-terphenyl diphosphine ligand reported by Agapie and coworkers demonstrated an interesting H-atom migration from nickel metal to the central arene moiety in the ligand framework. ${ }^{10}$ Additionally, we previously reported an in depth investigation on a stilbene diphosphine ligand, $t \mathrm{PCH}=\mathrm{CHP}(t \mathrm{PCH}=\mathrm{CHP}=((\mathrm{E})-1,2-\mathrm{bis}(2$-di-isopropylphosphinephenyl)-ethene)), which also demonstrates H-atom reservoir behavior. ${ }^{11-15}$ This mode of cooperativity can be a valuable tool for a variety of bond activation processes. For example, our group also reported a palladium carbene, $\left(\mathrm{PC}\left(\mathrm{sp}^{2}\right) \mathrm{P}\right) \mathrm{Pd}\left(\mathrm{PR}_{3}\right)\left(\left(\mathrm{PC}\left(\mathrm{sp}^{2}\right) \mathrm{P}\right)=\right.$ bis$[2-$ (di-iso-propyl- phosphino)phenyl]methylene, $\mathrm{R}=\mathrm{Ph}, \mathrm{CH}_{3}$ ), which is competent in a variety of bond activation processes due to the hydrogen acceptor capabilities of the nucleophilic carbene moiety. $^{16-21}$

In addition to cooperative bond activation processes, hemilabile ligands have also been a source of interesting chemistry. ${ }^{2,22-25}$ The design of multidentate ligands possessing a labile interaction capable of dissociating in the presence of a substrate has led to enhanced reactivity of 
metal complexes. ${ }^{6,26}$ For example, Hessen and coworkers reported the catalyst, $\left[\left(\eta^{5}-\right.\right.$ $\left.\left.\mathrm{C}_{5} \mathrm{H}_{4} \mathrm{CMe}_{2} \mathrm{R}\right) \mathrm{TiCl}_{3}\right]\left(\mathrm{R}=\mathrm{CH}_{3}, \mathrm{Ph}\right) .{ }^{26}$ They discovered that replacing the noncoordinating methyl substituent with a labile arene moiety transformed the ethane dimerization catalyst into a trimerization catalyst. Along with the dissociation of the labile moiety, the re-association helps stabilize metal centers, which may otherwise decompose. ${ }^{27,28}$

We previously reported two supporting ligands containing an olefin as the central chelating moiety, $t \mathrm{PCH}=\mathrm{CHP}$ and $t \mathrm{PCMe}=\mathrm{CMeP} \quad(t \mathrm{PCMe}=\mathrm{CMeP}=(\mathrm{E})-2,3-$ bis$(2-\mathrm{di}-i s o-$ propylphosphinephenyl)-2-butene). ${ }^{11,15}$ We observed hemilabile behavior with group 8, 9, and 11 metals, as well as $\mathrm{H}$-atom donor and acceptor capabilities with group 10 metals. We became interested in studying the cis analogue of $t \mathrm{PCMe}=\mathrm{CMeP}$ in order to see if the strain in the backbone resulting upon coordination would influence the reactivity. Herein, we present a study of the metal ligand cooperation exhibited by 2,2'-bis(di-iso-propylphosphino)-cis-diphenyl-1,2dimethylethene, $c \mathrm{PCMe}=\mathrm{CMeP}$, and palladium.

\section{Experimental}

\subsection{Materials and Methods}

All manipulations of air and water sensitive compounds were performed under a dry nitrogen atmosphere using an MBraun drybox. Glassware, vials, and stirbars were dried in an oven at $120{ }^{\circ} \mathrm{C}$ overnight and evacuated for $12 \mathrm{~h}$ in the antechamber before being brought into the drybox. All solvents were dried by passing through a column of activated alumina, followed by storage over molecular sieves and sodium. Deuterated solvents were purchased from Cambridge Isotope Laboratories. $\mathrm{C}_{6} \mathrm{D}_{6}$ was dried by stirring over $\mathrm{CaH}_{2}$ followed by filtration. $\mathrm{CDCl}_{3}$, was dried over molecular sieves. $\left[\left\{(\mathrm{TMS}) \mathrm{CH}_{2}\right\}_{2} \mathrm{Pd}(\mathrm{COD})\right]^{29}$ was prepared according to 
literature procedures. All other chemicals were commercially available and used as received unless otherwise indicated. NMR spectra were obtained on Bruker400 and Bruker500 spectrometers at ambient temperature. Chemical shift values are reported in ppm relative to residual internal protio solvents or to a TMS standard while using $\mathrm{CDCl}_{3}$ for ${ }^{1} \mathrm{H}$ and ${ }^{13} \mathrm{C}\{1 \mathrm{H}\}$ experiments. ${ }^{31} \mathrm{P}\left\{{ }^{1} \mathrm{H}\right\}$ NMR chemical shifts are reported relative to $\mathrm{H}_{3} \mathrm{PO}_{4}\left(85 \%\right.$ in $\mathrm{D}_{2} \mathrm{O}, 0$ ppm). Coupling constants are reported in $\mathrm{Hz}$.

\subsection{Synthesis of (Z)-2,3-Bis(2-di-iso-propylphosphinephenyl)-2- butene (cPCMe=CMeP, 1)}

The precursor (Z)-2,3-bis(2-bromophenyl)-2-butene was synthesized as previously reported in $90 \%$ yield. ${ }^{15,30}$ The precursor $(2.8 \mathrm{~g}, 7.3 \mathrm{mmol})$ was dissolved in $30 \mathrm{~mL}$ of $\mathrm{Et}_{2} \mathrm{O}$, and chilled to $-78{ }^{\circ} \mathrm{C}$. Two equivalents of $n$-butyllithium was added to the solution via syringe $(9.9$ $\mathrm{mL}, 15.8 \mathrm{mmol}$ ). The mixture was stirred at room temperature for $1 \mathrm{~h}$. The solution was once again chilled to $-78{ }^{\circ} \mathrm{C}$ before adding two equivalents of di-iso-propyl phosphine chloride (2.3 $\mathrm{mL}, 15.8 \mathrm{mmol})$. After stirring the solution for $12 \mathrm{~h}$, the mixture was quenched with $2 \mathrm{~mL}$ of a degassed $10 \%$ solution of $\mathrm{NH}_{4} \mathrm{Cl}$. The solution was dried over $\mathrm{Na}_{2} \mathrm{SO}_{4}$ followed by filtration over a pad of Celite. The volatiles were removed under reduced pressure, leaving behind the crude residue of $\mathbf{1}$. The compound was recrystalized from a concentrated solution of $n$-pentane in $83 \%$ yield. For 1: ${ }^{1} \mathrm{H}$ NMR $\left(400 \mathrm{MHz}, \mathrm{C}_{6} \mathrm{D}_{6}\right) \delta: 1.03\left(\mathrm{~m}, 12 \mathrm{H}, \mathrm{CH}\left(\mathrm{CH}_{3}\right)_{2}\right), 1.18(\mathrm{~m}, 12 \mathrm{H}$, $\left.\mathrm{CH}\left(\mathrm{CH}_{3}\right)_{2}\right), 1.80\left(\mathrm{~m}, 2 \mathrm{H}, \mathrm{CH}\left(\mathrm{CH}_{3}\right)_{2}\right), 2.05\left(\mathrm{~m}, 2 \mathrm{H}, \mathrm{CH}\left(\mathrm{CH}_{3}\right)_{2}\right), 2.25\left(\mathrm{~s}, 6 \mathrm{H}, \mathrm{CH}_{3} \mathrm{C}=\mathrm{CCH}_{3}\right), 6.87$ $\left(\mathrm{td}, J_{\mathrm{HH}}=4 \mathrm{~Hz}, J_{\mathrm{HP}}=3 \mathrm{~Hz}, 2 \mathrm{H}, \operatorname{Ar} H\right), 6.91\left(\mathrm{t}, J_{\mathrm{HH}}=8 \mathrm{~Hz}, 2 \mathrm{H}, \operatorname{Ar} H\right), 7.21\left(\mathrm{ddd}, J_{\mathrm{HH}}=8 \mathrm{~Hz}, J_{\mathrm{HP}}\right.$ $\left.=1 \mathrm{~Hz}, J_{\mathrm{HP}}=2 \mathrm{~Hz}, 2 \mathrm{H}, \operatorname{Ar} H\right), 7.49\left(\mathrm{dd}, J_{\mathrm{HH}}=7.5 \mathrm{~Hz}, J_{\mathrm{HP}}=3.5 \mathrm{~Hz}, 2 \mathrm{H}, \operatorname{Ar} H\right) .{ }^{31} \mathrm{P}\left\{{ }^{1} \mathrm{H}\right\} \mathrm{NMR}$ $\left(162 \mathrm{MHz}, \mathrm{C}_{6} \mathrm{D}_{6}\right) \delta:-1.86(\mathrm{~s}) .{ }^{13} \mathrm{C}\left\{{ }^{1} \mathrm{H}\right\}$ NMR $\left(100 \mathrm{MHz}, \mathrm{C}_{6} \mathrm{D}_{6}\right) \delta: 20.05\left(\mathrm{~d}, J_{\mathrm{CP}}=13 \mathrm{~Hz}\right.$, $\left.\mathrm{CH}\left(\mathrm{CH}_{3}\right)_{2}\right), 20.95\left(\mathrm{~d}, J_{\mathrm{CP}}=21 \mathrm{~Hz}, \mathrm{CH}\left(\mathrm{CH}_{3}\right)_{2}\right), 21.79\left(\mathrm{~d}, J_{\mathrm{CP}}=15 \mathrm{~Hz}, \mathrm{CH}\left(\mathrm{CH}_{3}\right)_{2}\right), 21.79\left(\mathrm{~d}, J_{\mathrm{CP}}=\right.$ 
$\left.15 \mathrm{~Hz}, \mathrm{CH}\left(\mathrm{CH}_{3}\right)_{2}\right), 24.08\left(\mathrm{~d}, J_{\mathrm{CP}}=10 \mathrm{~Hz}, \mathrm{CH}_{3} \mathrm{C}=\mathrm{CCH}_{3}\right), 24.83\left(\mathrm{~d}, J_{\mathrm{CP}}=14 \mathrm{~Hz}, \mathrm{CH}\left(\mathrm{CH}_{3}\right)_{2}\right)$, $27.63\left(\mathrm{~d}, J_{\mathrm{CP}}=16 \mathrm{~Hz}, \mathrm{CH}\left(\mathrm{CH}_{3}\right)_{2}\right), 126.0(\mathrm{~s}, \mathrm{ArC}), 128.46(\mathrm{~s}, \mathrm{ArC}), 131.60\left(\mathrm{dd}, J_{\mathrm{CP}}=8 \mathrm{~Hz}, J_{\mathrm{CP}}=\right.$ $4 \mathrm{~Hz}, \mathrm{ArC}), 131.97\left(\mathrm{~d}, J_{\mathrm{CP}}=3 \mathrm{~Hz}, \mathrm{ArC}\right), 134.50\left(\mathrm{~d}, J_{\mathrm{CP}}=6 \mathrm{~Hz}, \mathrm{CH}_{3} C=\mathrm{CCH}_{3}\right), 135.98\left(\mathrm{~d}, J_{\mathrm{CP}}=\right.$ $19 \mathrm{~Hz}, \operatorname{ArC}), 152.98\left(\mathrm{~d}, J_{\mathrm{CP}}=32 \mathrm{~Hz}, \operatorname{ArC}\right)$.

\subsection{Synthesis of $[(\mathrm{cPCMe}=\mathrm{CMeP}) \mathrm{Pd}]$ (2)}

A mixture of $c \mathrm{PCMe}=\mathrm{CMeP}(50 \mathrm{mg}, 0.11 \mathrm{mmol})$ and $\left[\left\{(\mathrm{TMS}) \mathrm{CH}_{2}\right\}_{2} \mathrm{Pd}(\mathrm{COD})\right](35.3 \mathrm{mg}$, $0.11 \mathrm{mmol}$ ) was stirred in $\mathrm{Et}_{2} \mathrm{O}$ at room temperature for $1 \mathrm{~h}$. The volatiles were removed under reduced pressure and the crude residue dissolved in $n$-pentane. The solution was filtered over Celite and cooled to $-35^{\circ} \mathrm{C}$ to induce crystallization. The final product, $(c \mathrm{PCMe}=\mathrm{CMeP}) \mathrm{Pd}(2)$, was isolated in $64 \%$ yield. The compound was stored at $-35{ }^{\circ} \mathrm{C}$ and kept in the dark to prevent backbone isomerization. For 2: ${ }^{1} \mathrm{H}$ NMR $\left(400 \mathrm{MHz}, \mathrm{C}_{6} \mathrm{D}_{6}\right) \delta: 0.81\left(\operatorname{app~q}, J_{\mathrm{HP}}=12 \mathrm{~Hz}, J_{\mathrm{HH}}=4\right.$ $\left.\mathrm{Hz}, 6 \mathrm{H}, \mathrm{CH}\left(\mathrm{CH}_{3}\right)_{2}\right), 1.18\left(\right.$ app q, $\left.J_{\mathrm{HP}}=8 \mathrm{~Hz}, J_{\mathrm{HH}}=4 \mathrm{~Hz}, 6 \mathrm{H}, \mathrm{CH}\left(\mathrm{CH}_{3}\right)_{2}\right), 1.30(\mathrm{~m}, 12 \mathrm{H}$, $\left.\mathrm{CH}\left(\mathrm{CH}_{3}\right)_{2}\right), 2.21\left(\mathrm{v} \mathrm{t}, 6 \mathrm{H},\left(\mathrm{CH}_{3}\right) \mathrm{C}=\mathrm{C}\left(\mathrm{CH}_{3}\right), 2.24\left(\mathrm{~m}, 4 \mathrm{H}, \mathrm{CH}\left(\mathrm{CH}_{3}\right)_{2}, 6.85\left(\mathrm{t}, J_{\mathrm{HH}}=8 \mathrm{~Hz}, 2 \mathrm{H}\right.\right.\right.$, $\operatorname{Ar} H), 7.0\left(\mathrm{t}, J_{\mathrm{HH}}=8 \mathrm{~Hz}, 2 \mathrm{H}, \operatorname{Ar} H\right), 7.06\left(\mathrm{~d}, J_{\mathrm{HH}}=10 \mathrm{~Hz}, 2 \mathrm{H}, \operatorname{Ar} H\right), 7.32\left(\mathrm{~d}, J_{\mathrm{HH}}=10 \mathrm{~Hz}, \operatorname{Ar} H\right)$. ${ }^{31} \mathrm{P}\left\{{ }^{1} \mathrm{H}\right\}$ NMR (200 MHz, $\left.\mathrm{C}_{6} \mathrm{D}_{6}\right) \delta: 48.64$ (s). ${ }^{13} \mathrm{C}\left\{{ }^{1} \mathrm{H}\right\}$ NMR (126 MHz, $\left.\mathrm{C}_{6} \mathrm{D}_{6}\right) \delta: 18.89$ (s, $\left.\mathrm{CH}\left(\mathrm{CH}_{3}\right)_{2}\right), 20.62\left(\mathrm{t}, J_{\mathrm{CP}}=6 \mathrm{~Hz}, \mathrm{CH}\left(\mathrm{CH}_{3}\right)_{2}\right), 20.67\left(\mathrm{t}, J_{\mathrm{CP}}=10 \mathrm{~Hz}, \mathrm{CH}\left(\mathrm{CH}_{3}\right)_{2}\right), 21.39(\mathrm{~s}$, $\left.\mathrm{CH}\left(\mathrm{CH}_{3}\right)_{2}\right), 22.32\left(\mathrm{t}, J_{\mathrm{CP}}=6 \mathrm{~Hz}, \mathrm{CH}\left(\mathrm{CH}_{3}\right)_{2}\right), 28.75\left(\mathrm{~s}, \mathrm{CH}\left(\mathrm{CH}_{3}\right)_{2}\right), 29.01\left(\mathrm{t}, J_{\mathrm{CP}}=5 \mathrm{~Hz}\right.$, $\mathrm{CH}_{3} \mathrm{C}=\mathrm{CCH}_{3}$ ), $92.97\left(\mathrm{t}, J_{\mathrm{CP}}=9 \mathrm{~Hz}, \mathrm{CH}_{3} C=\mathrm{CCH}_{3}\right), 125.33(\mathrm{~s}, \mathrm{ArC}), 128.97(\mathrm{~s}, \mathrm{ArC}), 129.48$ (t, $\left.J_{\mathrm{CP}}=8 \mathrm{~Hz}, \operatorname{ArC}\right), 132.15(\mathrm{~s}, \operatorname{ArC}), 146.24\left(\mathrm{t}, J_{\mathrm{CP}}=16 \mathrm{~Hz}, \operatorname{Ar} C\right), 155.65\left(\mathrm{t}, J_{\mathrm{CP}}=16 \mathrm{~Hz}, \operatorname{ArC}\right)$. Anal. Calcd. for $\mathrm{C}_{28} \mathrm{H}_{42} \mathrm{P}_{2} \mathrm{Pd}$ : C, 61.48; H, 7.74. Found: C, $61.56 \mathrm{H}, 7.83$. 


\subsection{Synthesis of $\left[(\mathrm{cPCMe}=\mathrm{CMeP}) \mathrm{PdCl} \mathrm{l}_{2}\right](\mathbf{3})$}

A THF solution of $c \mathrm{PCMe}=\mathrm{CMeP}(25 \mathrm{mg}, 0.06 \mathrm{mmol})$ was added to [(COD)PdCl 2$](16.2$ $\mathrm{mg}, 0.06 \mathrm{mmol}$ ) in a Schlenk tube and heated to $65^{\circ} \mathrm{C}$. After $1 \mathrm{~h}$, the volatiles were removed under reduced pressure and the resulting orange powder was triturated with $n$-pentane. The residual solid was dried under vacuum. Yield $32.9 \mathrm{mg}, 94 \%$. For 3: ${ }^{1} \mathrm{H} \mathrm{NMR}\left(500 \mathrm{MHz}, \mathrm{CDCl}_{3}\right)$ $\delta: 1.24\left(\operatorname{app~q}, 6 \mathrm{H}, J_{\mathrm{HP}}=10 \mathrm{~Hz}, \mathrm{CH}\left(\mathrm{CH}_{3}\right)_{2}\right), 1.32\left(\operatorname{app~q}, 6 \mathrm{H}, J_{\mathrm{HP}}=5 \mathrm{~Hz}, \mathrm{CH}\left(\mathrm{CH}_{3}\right)_{2}\right), 1.39(\operatorname{app~q}$, $\left.6 \mathrm{H}, J_{\mathrm{HP}}=10 \mathrm{~Hz}, \mathrm{CH}\left(\mathrm{CH}_{3}\right)_{2}\right), 1.59\left(\operatorname{app~q}, 6 \mathrm{H}, J_{\mathrm{HP}}=10 \mathrm{~Hz}\right), 1.89\left(\mathrm{~s}, 6 \mathrm{H}, \mathrm{CH}_{3} \mathrm{C}=\mathrm{CCH}_{3}\right), 3.08(\mathrm{~m}$, 4H, $\left.\mathrm{CH}\left(\mathrm{CH}_{3}\right)_{2}\right), 7.69(\mathrm{~m}, 4 \mathrm{H}, \mathrm{ArH}), 7.84\left(\mathrm{~d}, 2 \mathrm{H}, J_{\mathrm{HH}}=10 \mathrm{~Hz}, \mathrm{ArH}\right), 7.94\left(\mathrm{t}, 2 \mathrm{H}, J_{\mathrm{HH}}=5 \mathrm{~Hz}\right.$, $\mathrm{ArH}) .{ }^{31} \mathrm{P}\left\{{ }^{1} \mathrm{H}\right\}$ NMR $\left(200 \mathrm{MHz}, \mathrm{CDCl}_{3}\right) \delta: 68.7$ (s). ${ }^{13} \mathrm{C}\left\{{ }^{1} \mathrm{H}\right\}$ NMR (126 MHz, $\left.\mathrm{CDCl}_{3}\right) \delta: 17.25$ (s, $\left.\mathrm{CH}\left(\mathrm{CH}_{3}\right)_{2}\right), 18.34\left(\mathrm{~s}, \mathrm{CH}\left(\mathrm{CH}_{3}\right)_{2}\right), 19.48\left(\mathrm{~s}, \mathrm{CH}\left(\mathrm{CH}_{3}\right)_{2}\right), 19.80\left(\mathrm{~s}, \mathrm{CH}\left(\mathrm{CH}_{3}\right)_{2}\right), 26.48\left(\mathrm{t}, J_{\mathrm{CP}}=\right.$ $\left.13 \mathrm{~Hz}, \mathrm{CH}\left(\mathrm{CH}_{3}\right)_{2}\right), 126.88\left(\mathrm{t}, J_{\mathrm{CP}}=13 \mathrm{~Hz}, \mathrm{CH}\left(\mathrm{CH}_{3}\right)_{2}\right), 28.32\left(\mathrm{~s}, \mathrm{CH}_{3} \mathrm{C}=\mathrm{CCH}_{3}\right), 128.77\left(\mathrm{t}, J_{\mathrm{CP}}=9\right.$ $\mathrm{Hz}, \mathrm{ArC}), 129.19\left(\mathrm{~s}, \mathrm{CH}_{3} C=\mathrm{CCH}_{3}\right), 129.29\left(\mathrm{t}, J_{\mathrm{CP}}=20 \mathrm{~Hz}, \mathrm{ArC}\right), 130.23\left(\mathrm{t}, J_{\mathrm{CP}}=3 \mathrm{~Hz}, \operatorname{Ar} C\right.$ ), $132.30(\mathrm{~s}, \operatorname{ArC}), 135.20(\mathrm{~s}, \mathrm{ArC}), 150.42$ (t, $J_{\mathrm{CP}}=11 \mathrm{~Hz}, \mathrm{ArC}$ ). Anal. Calcd. for $\mathrm{C}_{28} \mathrm{H}_{42} \mathrm{Cl}_{2} \mathrm{P}_{2} \mathrm{Pd}$ : C, 54.43; H, 6.85. Found: C, $54.31 \mathrm{H}, 6.43$.

\subsection{Synthesis of $\left[(\mathrm{cPCMe}=\mathrm{CMeP}) \mathrm{PdI}\left(\mathrm{CH}_{3}\right)\right](4)$}

To a THF solution of $[(c \mathrm{PCMe}=\mathrm{CMeP}) \mathrm{Pd}](2,22.6 \mathrm{mg}, 0.04 \mathrm{mmol})$ was added $2.6 \mu \mathrm{L}$ $\mathrm{CH}_{3} \mathrm{I}(0.04 \mathrm{mmol})$ and the mixture was stirred for $1 \mathrm{~h}$. The volatiles were removed under reduced pressure and the resulting crude residue was triturated with $n$-pentane resulting in a pale yellow powder. The product, 4, was isolated in 65\% (12.9 mg) yield. For 4: ${ }^{1} \mathrm{H}$ NMR (400 MHz, $\left.\mathrm{C}_{6} \mathrm{D}_{6}\right)$ $\delta:-0.10\left(\mathrm{t}, 3 \mathrm{H}, J_{\mathrm{HP}}=8 \mathrm{~Hz}, \mathrm{PdCH}_{3}\right), 0.91\left(\operatorname{app~q}, 6 \mathrm{H} J_{\mathrm{HP}}=8 \mathrm{~Hz}, \mathrm{CH}\left(\mathrm{CH}_{3}\right)_{2}\right), 1.26(\mathrm{~m}, 6 \mathrm{H}$, $\left.\mathrm{CH}\left(\mathrm{CH}_{3}\right)_{2}\right), 1.50\left(\operatorname{app~q}, 6 \mathrm{H}, J_{\mathrm{HP}}=8 \mathrm{~Hz}, \mathrm{CH}\left(\mathrm{CH}_{3}\right)_{2}\right), 1.75\left(\right.$ app q, 6H, $\left.J_{\mathrm{HP}}=8 \mathrm{~Hz}, \mathrm{CH}\left(\mathrm{CH}_{3}\right)_{2}\right)$, $2.19\left(\mathrm{~s}, 6 \mathrm{H}, \mathrm{CH}_{3} \mathrm{C}=\mathrm{CCH}_{3}\right), 2.49\left(\mathrm{~m}, 2 \mathrm{H}, \mathrm{CH}\left(\mathrm{CH}_{3}\right)_{2}\right), 3.72\left(\mathrm{~m}, 2 \mathrm{H}, \mathrm{CH}\left(\mathrm{CH}_{3}\right)_{2}\right), 6.8\left(\mathrm{t}, 2 \mathrm{H}, J_{\mathrm{HH}}=8\right.$ 
$\mathrm{Hz}, \operatorname{Ar} H), 6.97\left(\mathrm{t}, 2 \mathrm{H}, J_{\mathrm{HH}}=8 \mathrm{~Hz}, \operatorname{Ar} H\right), 7.15(\mathrm{~m}, 2 \mathrm{H}, \operatorname{Ar} H), 7.28(\mathrm{~m}, 2 \mathrm{H}, \operatorname{Ar} H) .{ }^{31} \mathrm{P}\left\{{ }^{1} \mathrm{H}\right\} \mathrm{NMR}$ (162 MHz, $\left.\mathrm{C}_{6} \mathrm{D}_{6}\right) \delta: 36.24$ (s). ${ }^{13} \mathrm{C}\left\{{ }^{1} \mathrm{H}\right\} \mathrm{NMR}\left(100 \mathrm{MHz}, \mathrm{C}_{6} \mathrm{D}_{6}\right) \delta: 4.23$ (br s, $\left.\mathrm{PdCH}_{3}\right), 19.75$ (s, $\left.\mathrm{CH}\left(\mathrm{CH}_{3}\right)_{2}\right), 20.53\left(\mathrm{~s}, \mathrm{CH}\left(\mathrm{CH}_{3}\right)_{2}\right), 20.60\left(\mathrm{~s}, \mathrm{CH}\left(\mathrm{CH}_{3}\right)_{2}\right), 24.49\left(\mathrm{t}, J_{\mathrm{CP}}=9 \mathrm{~Hz}, \mathrm{CH}\left(\mathrm{CH}_{3}\right)_{2}\right), 24.96(\mathrm{t}$, $\left.J_{\mathrm{CP}}=9 \mathrm{~Hz}, \mathrm{CH}\left(\mathrm{CH}_{3}\right)_{2}\right), 26.08\left(\mathrm{~s}, \mathrm{CH}_{3} \mathrm{C}=\mathrm{CCH}_{3}\right), 29.82\left(\mathrm{t}, J_{\mathrm{CP}}=10 \mathrm{~Hz}, \mathrm{CH}\left(\mathrm{CH}_{3}\right)_{2}\right), 125.06(\mathrm{~s}$, $\operatorname{ArC}$ ), 128.06 (obscured by $\mathrm{C}_{6} \mathrm{D}_{6}, \operatorname{ArC}$ ), 130.91 (s, $\operatorname{ArC}$ ), 132.40 (t, $J_{\mathrm{CP}}=5 \mathrm{~Hz}, \operatorname{ArC}$ ), 135.99 (br $\left.\mathrm{s}, \mathrm{CH}_{3} \mathrm{C}=\mathrm{CCH}_{3}\right), 138.91\left(\mathrm{t}, J_{\mathrm{CP}}=16 \mathrm{~Hz}, \mathrm{ArC}\right), 149.47$ (s, ArC). Anal. Calcd. for $\mathrm{C}_{29} \mathrm{H}_{45} \mathrm{IP}_{2} \mathrm{Pd}: \mathrm{C}$, 50.56; H, 6.58. Found: C, 50.62 H, 6.40.

2.6 Synthesis of $\left[(c P C M e=C M e P) P d I\left(C_{6} H_{5}\right)\right](5)$

Iodobenzene $(5.7 \mu \mathrm{L}, 0.05 \mathrm{mmol})$ was added to a THF solution of $[(c \mathrm{PCMe}=\mathrm{CMeP}) \mathrm{Pd}]$ (2, $27.8 \mathrm{mg}, 0.05 \mathrm{mmol})$ and the resulting mixture was stirred for $1 \mathrm{~h}$. The volatiles were removed under reduced pressure and the crude residue was triturated with $n$-pentane. The product was recrystalized from a concentrated $\mathrm{Et}_{2} \mathrm{O}$ solution. Yield: $20.2 \mathrm{mg}, 0.04 \mathrm{mmol}, 72.8 \%$. For 5: ${ }^{1} \mathrm{H}$ NMR $\left(500 \mathrm{MHz}, \mathrm{C}_{6} \mathrm{D}_{6}\right) \delta: 0.83\left(\operatorname{app~q}, 6 \mathrm{H}, J_{\mathrm{HP}}=5 \mathrm{~Hz}, \mathrm{CH}\left(\mathrm{CH}_{3}\right)_{2}\right), 0.97(\operatorname{app~q}, 6 \mathrm{H}$, $\left.J_{\mathrm{HP}}=5 \mathrm{~Hz}, \mathrm{CH}\left(\mathrm{CH}_{3}\right)_{2}\right), 1.29\left(\mathrm{~m}, 2 \mathrm{H}, \mathrm{CH}\left(\mathrm{CH}_{3}\right)_{2}\right), 1.52\left(\operatorname{app~q}, 6 \mathrm{H}, J_{\mathrm{HP}}=10 \mathrm{~Hz}, \mathrm{CH}\left(\mathrm{CH}_{3}\right)_{2}\right), 1.70$ $\left(\operatorname{app~q}, 6 \mathrm{H}, J_{\mathrm{HP}}=10 \mathrm{~Hz}, \mathrm{CH}\left(\mathrm{CH}_{3}\right)_{2}\right), 2.20\left(\mathrm{~s}, 6 \mathrm{H}, \mathrm{CH}_{3} \mathrm{C}=\mathrm{CCH}_{3}\right), 3.81\left(\mathrm{~m}, 2 \mathrm{H}, \mathrm{CHCH}_{3}\right) 4.41(\mathrm{~d}$, $\left.1 \mathrm{H}, J_{\mathrm{HH}}=10 \mathrm{~Hz}, \operatorname{Ar} H\right), 6.18\left(\mathrm{t}, 1 \mathrm{H}, J_{\mathrm{HH}}=5 \mathrm{~Hz}, \operatorname{Ar} H\right), 6.67\left(\mathrm{t}, 1 \mathrm{H}, J_{\mathrm{HH}}=5 \mathrm{~Hz}, \operatorname{Ar} H\right) .6 .77(\mathrm{~m}$, 5H, ArH), $7.02\left(\mathrm{t}, J_{\mathrm{HH}}=5 \mathrm{~Hz}, \operatorname{Ar} H\right), 7.14$ (obscured by solvent, $\left.2 \mathrm{H}, \operatorname{Ar} H\right), 7.81\left(\mathrm{~d}, 1 \mathrm{H}, J_{\mathrm{HH}}=10\right.$ $\mathrm{Hz}, \operatorname{Ar} H) .{ }^{31} \mathrm{P}\left\{{ }^{1} \mathrm{H}\right\}$ NMR $\left(200 \mathrm{MHz}, \mathrm{C}_{6} \mathrm{D}_{6}\right) \delta: 32.39$ (s). ${ }^{13} \mathrm{C}\left\{{ }^{1} \mathrm{H}\right\}$ NMR $\left(126 \mathrm{MHz}, \mathrm{C}_{6} \mathrm{D}_{6}\right) \delta$ : $20.38\left(\mathrm{t}, J_{\mathrm{CP}}=3 \mathrm{~Hz}, \mathrm{CH}\left(\mathrm{CH}_{3}\right)_{2}\right), 20.56\left(\mathrm{t}, J_{\mathrm{CP}}=1 \mathrm{~Hz}, \mathrm{CH}\left(\mathrm{CH}_{3}\right)_{2}\right), 21.10\left(\mathrm{~s}, \mathrm{CH}\left(\mathrm{CH}_{3}\right)_{2}\right), 24.38(\mathrm{t}$, $\left.J_{\mathrm{CP}}=6 \mathrm{~Hz}, \mathrm{CH}\left(\mathrm{CH}_{3}\right)_{2}\right), 24.76\left(\mathrm{t}, J_{\mathrm{CP}}=10 \mathrm{~Hz}, \mathrm{CH}\left(\mathrm{CH}_{3}\right)_{2}\right), 25.36\left(\mathrm{~s}, \mathrm{CH}_{3} \mathrm{C}=\mathrm{CCH}_{3}\right), 30.51\left(\mathrm{t}, J_{\mathrm{CP}}\right.$ $\left.=11 \mathrm{~Hz}, \mathrm{CH}\left(\mathrm{CH}_{3}\right)_{2}\right), 122.88\left(\mathrm{~s}, \mathrm{CH}_{3} C=\mathrm{CCH}_{3}\right), 124.50(\mathrm{~s}, \mathrm{ArC}), 125.07\left(\mathrm{t}, J_{\mathrm{CP}}=3 \mathrm{~Hz}, \mathrm{ArC}\right)$, $126.10(\mathrm{~s}, \operatorname{ArC}), 127.97(\mathrm{~s}, \operatorname{ArC}), 128.35$ (s, $\operatorname{ArC}), 131.70(\mathrm{~s}, \operatorname{ArC}), 133.25\left(\mathrm{t}, J_{\mathrm{CP}}=5 \mathrm{~Hz}, \operatorname{Ar} C\right)$, 
$136.41\left(\mathrm{t}, J_{\mathrm{CP}}=18 \mathrm{~Hz}, \operatorname{ArC}\right), 137.30(\mathrm{~s}, \operatorname{ArC}), 138.05\left(\mathrm{t}, J_{\mathrm{CP}}=4 \mathrm{~Hz}, \operatorname{ArC}\right), 139.16\left(\mathrm{t}, J_{\mathrm{CP}}=4 \mathrm{~Hz}\right.$ $\mathrm{ArC}), 148.26\left(\mathrm{t}, J_{\mathrm{CP}}=8 \mathrm{~Hz}, \mathrm{ArC}\right.$ ). Anal. Calcd. for $\mathrm{C}_{34} \mathrm{H}_{47} \mathrm{IP}_{2} \mathrm{Pd}: \mathrm{C}, 54.38 ; \mathrm{H}, 6.31$. Found: $\mathrm{C}$, $54.45 \mathrm{H}, 6.35$.

\subsection{Synthesis of $\left[(c P C M e=C M e P) P d\left(\mathrm{SiH}_{2} \mathrm{Ph}_{2}\right)\right](\mathbf{6})$}

Diphenylsilane $(0.02 \mathrm{~mL}, 0.1 \mathrm{mmol})$ was added via syringe to a diethyl ether solution of $[(c \mathrm{PCMe}=\mathrm{CMeP}) \mathrm{Pd}](2,33.1 \mathrm{mg}, 0.06 \mathrm{mmol})$. The mixture was stirred at room temperature for $1 \mathrm{~h}$. The volatiles were removed under reduced pressure. The crude residue was dissolved in minimum amount of $n$-pentane and stored at $-35^{\circ} \mathrm{C}$. The product, $\left[(c \mathrm{PCMe}=\mathrm{CMeP}) \mathrm{Pd}\left(\mathrm{H}_{2} \mathrm{SiPh}_{2}\right)\right]$, crystalized from this concentrated solution in $48 \%$ yield $(21.2 \mathrm{mg})$. For $6:{ }^{1} \mathrm{H}$ NMR (400 MHz, $\left.\mathrm{C}_{6} \mathrm{D}_{6}\right) \delta: 0.85\left(\right.$ app q, 6H, $\left.J_{\mathrm{HP}}=8 \mathrm{~Hz}, \mathrm{CH}\left(\mathrm{CH}_{3}\right)_{2}\right), 1.19\left(\right.$ app q, 6H, $\left.J_{\mathrm{HP}}=4 \mathrm{~Hz}, \mathrm{CH}\left(\mathrm{CH}_{3}\right)_{2}\right), 1.28$ (app q, $\left.12 \mathrm{H}, J_{\mathrm{HP}}=8 \mathrm{~Hz}, \mathrm{CH}\left(\mathrm{CH}_{3}\right)_{2}\right), 2.15\left(\mathrm{vt}, 6 \mathrm{H}, \mathrm{CH}_{3} \mathrm{C}=\mathrm{CCH}_{3}\right), 2.21\left(\mathrm{~m}, 2 \mathrm{H}, \mathrm{CH}\left(\mathrm{CH}_{3}\right)_{2}\right), 2.32$ $\left(\mathrm{m}, 2 \mathrm{H}, \mathrm{CH}\left(\mathrm{CH}_{3}\right)_{2}\right), 4.55\left(\mathrm{~s}, 2 \mathrm{H}, J_{\mathrm{HSi}}=96 \mathrm{~Hz}, \mathrm{Si} H_{2} \mathrm{Ph}_{2}\right), 6.85\left(\mathrm{td}, J_{\mathrm{HH}}=8 \mathrm{~Hz}, J_{\mathrm{HP}}=4 \mathrm{~Hz}, \mathrm{Ar} H\right)$, $6.97\left(\mathrm{t}, J_{\mathrm{HH}}=8 \mathrm{~Hz}, \operatorname{Ar} H\right), 7.10(\mathrm{~m}, 2 \mathrm{H}, \mathrm{Ar} H), 7.15(\mathrm{~m}, 9 \mathrm{H}, \mathrm{Ar} H), 7.2\left(\mathrm{dd}, J_{\mathrm{HH}}=8 \mathrm{~Hz}, J_{\mathrm{HP}}=4 \mathrm{~Hz}\right.$,

ArH). ${ }^{31} \mathrm{P}\left\{{ }^{1} \mathrm{H}\right\} \operatorname{NMR}\left(162 \mathrm{MHz}, \mathrm{C}_{6} \mathrm{D}_{6}\right) \delta: 45.75(\mathrm{~s}) .{ }^{13} \mathrm{C}\left\{{ }^{1} \mathrm{H}\right\} \operatorname{NMR}\left(100 \mathrm{MHz}, \mathrm{C}_{6} \mathrm{D}_{6}\right) \delta: 19.28(\mathrm{~s}$, $\left.\mathrm{CH}\left(\mathrm{CH}_{3}\right)_{2}\right), 20.80\left(\mathrm{t}, J_{\mathrm{CP}}=6 \mathrm{~Hz}, \mathrm{CH}\left(\mathrm{CH}_{3}\right)_{2}\right), 21.40\left(\mathrm{t}, J_{\mathrm{CP}}=6 \mathrm{~Hz}, \mathrm{CH}\left(\mathrm{CH}_{3}\right)_{2}\right), 21.53\left(\mathrm{t}, J_{\mathrm{CP}}=5\right.$ $\left.\mathrm{Hz}, \mathrm{CH}\left(\mathrm{CH}_{3}\right)_{2}\right), 23.33\left(\right.$ br s, $\left.\mathrm{CH}\left(\mathrm{CH}_{3}\right)_{2}\right), 27.69\left(\mathrm{~s}, \mathrm{CH}_{3} \mathrm{C}=\mathrm{CCH}_{3}\right), 28.97$ (t, $J_{\mathrm{CP}}=6 \mathrm{~Hz}$, $\left.\mathrm{CH}\left(\mathrm{CH}_{3}\right)_{2}\right), 104.65$ (br s, $\mathrm{CH}_{3} C=\mathrm{CCH}_{3}$ ), 125.42 (s, $\mathrm{ArC}$ ), 128.20 (s, $\mathrm{ArC}$ ), 128.85 (s, $\mathrm{ArC}$ ), $129.52(\mathrm{~s}, \operatorname{ArC}), 130.01\left(\mathrm{t}, J_{\mathrm{CP}}=7 \mathrm{~Hz}, \operatorname{ArC}\right), 131.78(\mathrm{~s}, \operatorname{ArC}), 135.40($ br s, $\operatorname{ArC}), 136.26(\mathrm{~s}$, $\operatorname{ArC}$ ), 143.59 (br s, $\operatorname{ArC}$ ), $154.44\left(\mathrm{t}, J_{\mathrm{CP}}=5 \mathrm{~Hz}, \mathrm{ArC}\right.$ ). Anal. Calcd. for $\mathrm{C}_{40} \mathrm{H}_{54} \mathrm{P}_{2} \mathrm{PdSi}$ : C, 65.69; H, 7.44. Found: C, $66.21 \mathrm{H}, 7.89$. 


\subsection{Synthesis of $[(c P C M e=C M e P) P d H C l]$ (7)}

To a diethyl ether solution of $[(c \mathrm{PCMe}=\mathrm{CMeP}) \mathrm{Pd}](2,20.2 \mathrm{mg}, 0.04 \mathrm{mmol})$ was added $0.03 \mathrm{~mL}$ of a $1 \mathrm{M}$ ethereal $\mathrm{HCl}$ solution. The mixture was stirred for $1 \mathrm{~h}$. The volatiles were removed under reduced pressure and the resulting white powder was triturated with $n$-pentane.

The solid was dried under vacuum. Yield: $17.6 \mathrm{mg}, 82 \%$. For 7: ${ }^{1} \mathrm{H}$ NMR (500 MHz, $\left.\mathrm{C}_{6} \mathrm{D}_{6}\right) \delta$ : $14.58(\mathrm{~s}, 1 \mathrm{H}, \mathrm{Pd} H), 0.76\left(\right.$ app q, 6H, $\left.J_{\mathrm{HP}}=10 \mathrm{~Hz}, \mathrm{CH}\left(\mathrm{CH}_{3}\right)_{2}\right), 0.94\left(\right.$ app q, 6H, $J_{\mathrm{HP}}=10 \mathrm{~Hz}$, $\left.\mathrm{CH}\left(\mathrm{CH}_{3}\right)_{2}\right), 1.24\left(\right.$ app q, 6H, $\left.J_{\mathrm{HP}}=5 \mathrm{~Hz}, \mathrm{CH}\left(\mathrm{CH}_{3}\right)_{2}\right), 1.77$ (app q, 6H, $\left.J_{\mathrm{HP}}=10 \mathrm{~Hz}, \mathrm{CH}\left(\mathrm{CH}_{3}\right)_{2}\right)$, $2.16\left(\mathrm{~m}, 2 \mathrm{H}, \mathrm{CH}\left(\mathrm{CH}_{3}\right)_{2}\right), 2.31\left(\mathrm{~s}, 6 \mathrm{H}, \mathrm{CH}_{3} \mathrm{C}=\mathrm{CCH}_{3}\right), 2.81\left(\mathrm{~m}, 2 \mathrm{H}, \mathrm{CH}\left(\mathrm{CH}_{3}\right)_{2}\right), 6.77\left(\mathrm{t}, 2 \mathrm{H}, J_{\mathrm{HH}}=\right.$ $5 \mathrm{~Hz}, \operatorname{Ar} H), 6.91(\mathrm{~m}, 2 \mathrm{H}, \mathrm{Ar} H), 6.96\left(\mathrm{t}, 2 \mathrm{H}, J_{\mathrm{HH}}=5 \mathrm{~Hz}, \mathrm{Ar} H\right), 7.23\left(\mathrm{dd}, 2 \mathrm{H}, J_{\mathrm{HH}}=10 \mathrm{~Hz}, J_{\mathrm{HP}}=\right.$ $5 \mathrm{~Hz}) .{ }^{31} \mathrm{P}\left\{{ }^{1} \mathrm{H}\right\}$ NMR $\left(200 \mathrm{MHz}, \mathrm{C}_{6} \mathrm{D}_{6}\right) \delta: 37.28(\mathrm{~s}) .{ }^{13} \mathrm{C}\left\{{ }^{1} \mathrm{H}\right\}$ NMR $\left(100 \mathrm{MHz}, \mathrm{C}_{6} \mathrm{D}_{6}\right) \delta: 17.18(\mathrm{~s}$, $\left.\mathrm{CH}\left(\mathrm{CH}_{3}\right)_{2}\right), 21.10\left(\mathrm{~s}, \mathrm{CH}\left(\mathrm{CH}_{3}\right)_{2}\right), 21.13\left(\mathrm{~s}, \mathrm{CH}\left(\mathrm{CH}_{3}\right)_{2}\right), 21.21\left(\mathrm{~s}, \mathrm{CH}\left(\mathrm{CH}_{3}\right)_{2}\right), 22.08\left(\mathrm{t}, J_{\mathrm{CP}}=6 \mathrm{~Hz}\right.$, $\left.\mathrm{CH}\left(\mathrm{CH}_{3}\right)_{2}\right), 27.17\left(\mathrm{~s}, \mathrm{CH}_{3} \mathrm{C}=\mathrm{CCH}_{3}\right), 27.76\left(\mathrm{t}, J_{\mathrm{CP}}=9 \mathrm{~Hz}, \mathrm{CH}\left(\mathrm{CH}_{3}\right)_{2}\right), 124.58\left(\mathrm{t}, J_{\mathrm{CP}}=3 \mathrm{~Hz}\right)$, $129.05(\mathrm{~s}, \operatorname{ArC}), 131.36\left(\mathrm{t}, J_{\mathrm{CP}}=5 \mathrm{~Hz}, \operatorname{ArC}\right), 132.64(\mathrm{~s}, \operatorname{ArC}), 135.15\left(\mathrm{t}, J_{\mathrm{CP}}=3 \mathrm{~Hz}\right.$ $\left.\mathrm{CH}_{3} C=\mathrm{CCH}_{3}\right), 137.20\left(\mathrm{t}, J_{\mathrm{CP}}=19 \mathrm{~Hz}, \mathrm{ArC}\right), 152.44\left(\mathrm{t}, J_{\mathrm{CP}}=9 \mathrm{~Hz}, \mathrm{ArC}\right)$.

\subsection{Synthesis of [(PCMe-CHMeP)PdCl] (8)}

A benzene solution of $[(c \mathrm{PCMe}=\mathrm{CMeP}) \mathrm{PdHCl}](7,17.6 \mathrm{mg}, 0.03 \mathrm{mmol})$ was heated to $80{ }^{\circ} \mathrm{C}$ for $2 \mathrm{~h}$. The ${ }^{1} \mathrm{H}$ and ${ }^{31} \mathrm{P}\left\{{ }^{1} \mathrm{H}\right\}$ spectra of an aliquot showed clean conversion to 8 . The volatiles were removed under reduced pressure resulting in a yellow powder $(17.0 \mathrm{mg}, 96 \%)$. For 8: ${ }^{1} \mathrm{H}$ NMR $\left(500 \mathrm{MHz}, \mathrm{C}_{6} \mathrm{D}_{6}\right) \delta: 0.97\left(\mathrm{~m}, 6 \mathrm{H}, \mathrm{CH}\left(\mathrm{CH}_{3}\right)_{2}\right), 1.11\left(\mathrm{~m}, 6 \mathrm{H}, \mathrm{CH}\left(\mathrm{CH}_{3}\right)_{2}\right), 1.31(\mathrm{dd}, 3 \mathrm{H}$, $\left.J_{\mathrm{HP}}=20 \mathrm{~Hz}, J_{\mathrm{HH}}=5 \mathrm{~Hz}, \mathrm{CH}\left(\mathrm{CH}_{3}\right)_{2}\right), 1.47\left(\mathrm{~m}, 9 \mathrm{H}, \mathrm{CH}_{3}, \mathrm{CH}\left(\mathrm{CH}_{3}\right)_{2}\right), 1.54\left(\mathrm{~d}, 3 \mathrm{H}, J_{\mathrm{HH}}=5 \mathrm{~Hz}\right.$, $\left.\mathrm{CH}_{3}\right), 1.73\left(\mathrm{dd}, 3 \mathrm{H}, J_{\mathrm{HP}}=15 \mathrm{~Hz}, J_{\mathrm{HH}}=5 \mathrm{~Hz}\right), 2.42\left(\mathrm{~m}, 2 \mathrm{H}, \mathrm{CH}\left(\mathrm{CH}_{3}\right)_{2}\right), 2.92\left(\mathrm{~m}, 1 \mathrm{H}, \mathrm{CHCH}_{3}\right)$, $3.26\left(\mathrm{~m}, 1 \mathrm{H}, \mathrm{CH}\left(\mathrm{CH}_{3}\right)_{2}\right), 3.44\left(\mathrm{~m}, 1 \mathrm{H}, \mathrm{CH}\left(\mathrm{CH}_{3}\right)_{2}\right), 7.03(\mathrm{~m}, 2 \mathrm{H}, \mathrm{Ar} H), 7.17(\mathrm{~m}, 4 \mathrm{H}, \mathrm{ArH}), 7.29$ 
$\left(\mathrm{t}, 1 \mathrm{H}, J_{\mathrm{HH}}=5 \mathrm{~Hz}, \operatorname{Ar} H\right), 7.56\left(\mathrm{dd}, 1 \mathrm{H}, J_{\mathrm{HH}}=10 \mathrm{~Hz}, J_{\mathrm{HP}}=5 \mathrm{~Hz}, \operatorname{Ar} H\right) .{ }^{31} \mathrm{P}\left\{{ }^{1} \mathrm{H}\right\} \mathrm{NMR}(200 \mathrm{MHz}$, $\left.\mathrm{C}_{6} \mathrm{D}_{6}\right) \delta: 18.49\left(\mathrm{~d}, J_{P P}=356 \mathrm{~Hz}\right), 66.09\left(\mathrm{~d}, J_{P P}=356 \mathrm{~Hz}\right) .{ }^{13} \mathrm{C}\left\{{ }^{1} \mathrm{H}\right\} \mathrm{NMR}\left(100 \mathrm{MHz}, \mathrm{C}_{6} \mathrm{D}_{6}\right) \delta$ : $17.12\left(\mathrm{dd}, J_{\mathrm{CP}}=4 \mathrm{~Hz}, J_{\mathrm{CP}}=2 \mathrm{~Hz}, \mathrm{CH}\left(\mathrm{CH}_{3}\right)_{2}\right), 18.54\left(\mathrm{~s}, \mathrm{CH}\left(\mathrm{CH}_{3}\right)_{2}\right), 19.81\left(\mathrm{~s}, \mathrm{CH}\left(\mathrm{CH}_{3}\right)\right), 19.62$ $\left(\mathrm{d}, J_{\mathrm{CP}}=5 \mathrm{~Hz}, \mathrm{CH}\left(\mathrm{CH}_{3}\right)_{2}\right), 19.85\left(\mathrm{~d}, J_{\mathrm{CP}}=5 \mathrm{~Hz}, \mathrm{CH}\left(\mathrm{CH}_{3}\right)_{2}\right), 20.43\left(\mathrm{~d}, J_{\mathrm{CP}}=6 \mathrm{~Hz}, \mathrm{CH}\left(\mathrm{CH}_{3}\right)_{2}\right)$,

$21.20\left(\mathrm{~d}, J_{\mathrm{CP}}=6 \mathrm{~Hz}, \mathrm{CH}\left(\mathrm{CH}_{3}\right)_{2}\right), 22.11\left(\mathrm{~d}, J_{\mathrm{CP}}=7 \mathrm{~Hz}, \mathrm{CH}\left(\mathrm{CH}_{3}\right)_{2}\right), 24.16\left(\mathrm{~d}, J_{\mathrm{CP}}=21 \mathrm{~Hz}\right.$, $\left.\mathrm{CH}\left(\mathrm{CH}_{3}\right)_{2}\right), 25.40\left(\mathrm{dd}, J_{\mathrm{CP}}=23 \mathrm{~Hz}, J_{\mathrm{CP}}=2 \mathrm{~Hz}, \mathrm{CH}\left(\mathrm{CH}_{3}\right)_{2}\right), 26.81\left(\mathrm{dd}, J_{\mathrm{CP}}=22 \mathrm{~Hz}, J_{\mathrm{CP}}=3 \mathrm{~Hz}\right.$, $\left.C \mathrm{H}\left(\mathrm{CH}_{3}\right)_{2}\right), 27.28\left(\mathrm{~d}, J_{\mathrm{CP}}=3 \mathrm{~Hz}, \mathrm{CH}\left(\mathrm{CH}_{3}\right)_{2}\right), 27.48\left(\mathrm{~d}, J_{\mathrm{CP}}=3 \mathrm{~Hz}, \mathrm{CH}\left(\mathrm{CH}_{3}\right)_{2}\right), 36.33\left(\mathrm{dd}, J_{\mathrm{CP}}=\right.$ $5 \mathrm{~Hz}, J_{\mathrm{CP}}=1 \mathrm{~Hz}, \operatorname{PdC}\left(\mathrm{CH}_{3}\right), 55.45\left(\mathrm{~d}, J_{\mathrm{CP}}=11 \mathrm{~Hz}, \mathrm{CH}\left(\mathrm{CH}_{3}\right)\right), 60.99\left(\mathrm{~d}, J_{\mathrm{CP}}=7 \mathrm{~Hz}, \operatorname{PdC}\left(\mathrm{CH}_{3}\right)\right)$, $126.35\left(\mathrm{~d}, J_{\mathrm{CP}}=20 \mathrm{~Hz}, \operatorname{Ar} C\right), 126.57\left(\mathrm{~d}, J_{\mathrm{CP}}=5 \mathrm{~Hz}, \operatorname{Ar} C\right), 126.97\left(\mathrm{~d}, J_{\mathrm{CP}}=5 \mathrm{~Hz}, \operatorname{Ar} C\right), 130.93$ $\left(\mathrm{d}, J_{\mathrm{CP}}=3 \mathrm{~Hz}, \mathrm{ArC}\right), 131.21\left(\mathrm{~d}, J_{\mathrm{CP}}=2 \mathrm{~Hz}, \mathrm{ArC}\right), 131.78\left(\mathrm{~d}, J_{\mathrm{CP}}=3 \mathrm{~Hz}, \mathrm{ArC}\right), 133.47\left(\mathrm{~d}, J_{\mathrm{CP}}=\right.$ $3 \mathrm{~Hz}, \operatorname{ArC}), 133.53\left(\mathrm{~d}, J_{\mathrm{CP}}=3 \mathrm{~Hz}, \operatorname{ArC}\right), 156.29$ (s, $\left.\operatorname{ArC}\right), 156.49$ (s, $\left.\operatorname{ArC}\right), 169.67(\mathrm{~s}, \operatorname{ArC})$, 170.05 (s, ArC). Anal. Calcd. for $\mathrm{C}_{28} \mathrm{H}_{43} \mathrm{ClP}_{2} \mathrm{Pd}$ : C, 57.64; H, 7.43. Found: C, 57.39 H, 7.32.

2.10 Synthesis of $\left[\left(\mathrm{PCMe}-\mathrm{C}\left(=\mathrm{CH}_{2}\right) \mathrm{P}\right) \mathrm{PdCl}\right]$ (9)

Benzyl potassium $(5.3 \mathrm{mg}, \quad 0.04 \mathrm{mmol})$ was added to a THF solution of $\left[(c \mathrm{PCMe}=\mathrm{CMeP}) \mathrm{PdCl}_{2}\right](3,25 \mathrm{mg}, 0.04 \mathrm{mmol})$. The mixture was allowed to stir for $1 \mathrm{~h}$. The volatiles were removed under reduced pressure and the crude residue was dissolved in a minimum amount of $n$-pentane. The product crystalized at $-35^{\circ} \mathrm{C}$ in $77 \%$ yield $(18.1 \mathrm{mg})$. For $\mathbf{9}$ : ${ }^{1} \mathrm{H}$ NMR $\left(400 \mathrm{MHz}, \mathrm{C}_{6} \mathrm{D}_{6}\right) \delta: 0.84\left(\mathrm{dd}, 3 \mathrm{H}, J_{\mathrm{HP}}=12 \mathrm{~Hz}, J_{\mathrm{HH}}=4 \mathrm{~Hz}, \mathrm{CH}\left(\mathrm{CH}_{3}\right)_{2}\right), 1.16(\mathrm{~m}, 12 \mathrm{H}$, $\left.\mathrm{CH}\left(\mathrm{CH}_{3}\right)_{2}\right), 1.42\left(\mathrm{dd}, 3 \mathrm{H}, J_{\mathrm{HP}}=20 \mathrm{~Hz}, J_{\mathrm{HH}}=8 \mathrm{~Hz}, \mathrm{CH}\left(\mathrm{CH}_{3}\right)_{2}\right), 1.50\left(\mathrm{dd}, 3 \mathrm{H}, J_{\mathrm{HP}}=8 \mathrm{~Hz}, J_{\mathrm{HP}}=4\right.$ $\left.\mathrm{Hz}, \mathrm{CH}_{3} \mathrm{CPd}\right), 1.55\left(\mathrm{dd}, 3 \mathrm{H}, J_{\mathrm{HP}}=20 \mathrm{~Hz}, J_{\mathrm{HH}}=4 \mathrm{~Hz}, \mathrm{CH}\left(\mathrm{CH}_{3}\right)_{2}\right), 1.73\left(\mathrm{dd}, 3 \mathrm{H}, J_{\mathrm{HP}}=20 \mathrm{~Hz}, J_{\mathrm{HH}}\right.$ $\left.=5 \mathrm{~Hz}, \mathrm{CH}\left(\mathrm{CH}_{3}\right)_{2}\right), 2.43\left(\mathrm{~m}, 2 \mathrm{H}, \mathrm{CH}\left(\mathrm{CH}_{3}\right)_{2}\right), 3.05\left(\mathrm{~m}, 1 \mathrm{H}, \mathrm{CH}\left(\mathrm{CH}_{3}\right)_{2}\right), 3.39\left(\mathrm{~m}, 1 \mathrm{H}, \mathrm{CH}\left(\mathrm{CH}_{3}\right)_{2}\right)$, $4.63\left(\mathrm{dd}, 1 \mathrm{H}, J_{\mathrm{HP}}=3 \mathrm{~Hz}, J_{\mathrm{HH}}=2 \mathrm{~Hz}, \mathrm{C}=\mathrm{C} H_{2}\right), 4.85\left(\mathrm{brdd}, 1 \mathrm{H}, J_{\mathrm{HP}}=8 \mathrm{~Hz}, J_{\mathrm{HH}}=4 \mathrm{~Hz}, \mathrm{C}=\mathrm{CH}_{2}\right)$, 
$7.02\left(\mathrm{t}, 1 \mathrm{H}, J_{\mathrm{HH}}=4 \mathrm{~Hz}, \operatorname{Ar} H\right), 7.14(\mathrm{~m}, 2 \mathrm{H}, \operatorname{Ar} H), 7.22(\mathrm{~m}, 3 \mathrm{H}, \operatorname{Ar} H), 7.38\left(\mathrm{dd}, 2 \mathrm{H}, J_{\mathrm{HH}}=8 \mathrm{~Hz}\right.$ $\left.J_{\mathrm{HP}}=4 \mathrm{~Hz}, \operatorname{Ar} H\right), 7.51\left(\mathrm{~d}, 2 \mathrm{H}, J_{\mathrm{HH}}=4 \mathrm{~Hz}, \operatorname{Ar} H\right) .{ }^{31} \mathrm{P}\left\{{ }^{1} \mathrm{H}\right\} \mathrm{NMR}\left(200 \mathrm{MHz}, \mathrm{C}_{6} \mathrm{D}_{6}\right) \delta: 19.84, J_{\mathrm{PP}}=$ $356 \mathrm{~Hz}), 65.80\left(\mathrm{~d}, J_{\mathrm{PP}}=356 \mathrm{~Hz}\right) .{ }^{13} \mathrm{C}\left\{{ }^{1} \mathrm{H}\right\} \mathrm{NMR}\left(126 \mathrm{MHz}, \mathrm{C}_{6} \mathrm{D}_{6}\right) \delta: 16.27\left(\mathrm{dd}, J_{\mathrm{CP}}=6 \mathrm{~Hz}, J_{\mathrm{CP}}=\right.$ $\left.4 \mathrm{~Hz}, \mathrm{CH}\left(\mathrm{CH}_{3}\right)_{2}\right), 17.71\left(\mathrm{t}, J_{\mathrm{CP}}=4 \mathrm{~Hz}, \mathrm{CH}\left(\mathrm{CH}_{3}\right)_{2}\right), 19.12\left(\mathrm{~d}, J_{\mathrm{CP}}=5 \mathrm{~Hz}, \mathrm{CH}\left(\mathrm{CH}_{3}\right)_{2}\right), 19.32(\mathrm{~d}$, $\left.J_{\mathrm{CP}}=5 \mathrm{~Hz}, \mathrm{CH}\left(\mathrm{CH}_{3}\right)_{2}\right), 19.59\left(\mathrm{dd}, J_{\mathrm{CP}}=4 \mathrm{~Hz}, J_{\mathrm{CP}}=1 \mathrm{~Hz}, \mathrm{CH}\left(\mathrm{CH}_{3}\right)_{2}\right), 19.68\left(\mathrm{~d}, J_{\mathrm{CP}}=5 \mathrm{~Hz}\right.$, $\left.\mathrm{CH}\left(\mathrm{CH}_{3}\right)_{2}\right), 19.77\left(\mathrm{~d}, J_{\mathrm{CP}}=6 \mathrm{~Hz}, \mathrm{CH}\left(\mathrm{CH}_{3}\right)_{2}\right), 20.48\left(\mathrm{~d}, J_{\mathrm{CP}}=9 \mathrm{~Hz}, \mathrm{CH}\left(\mathrm{CH}_{3}\right)_{2}\right), 24.30\left(\mathrm{dd}, J_{\mathrm{CP}}=\right.$ $\left.20 \mathrm{H}, J_{\mathrm{CP}}=3 \mathrm{~Hz}, \mathrm{CH}\left(\mathrm{CH}_{3}\right)_{2}\right), 25.03\left(\mathrm{dd}, J_{\mathrm{CP}}=18 \mathrm{~Hz}, J_{\mathrm{CP}}=1 \mathrm{~Hz}, \mathrm{CH}\left(\mathrm{CH}_{3}\right)_{2}\right), 25.12(\mathrm{br} \mathrm{s}$, $\left.\mathrm{CH}_{3} \mathrm{CPd}\right), 25.18\left(\mathrm{dd}, J_{\mathrm{CP}}=20 \mathrm{~Hz}, J_{\mathrm{CP}}=3 \mathrm{~Hz}, \mathrm{CH}\left(\mathrm{CH}_{3}\right)_{2}\right), 34.30\left(\mathrm{dd}, J_{\mathrm{CP}}=5 \mathrm{~Hz}, J_{\mathrm{CP}}=1 \mathrm{~Hz}\right.$, $\left.C=\mathrm{CH}_{2}\right), 112.73\left(\mathrm{~d}, J_{\mathrm{CP}}=5 \mathrm{~Hz}, \mathrm{C}=\mathrm{CH}_{2}\right), 126.80\left(\mathrm{~d}, J_{\mathrm{CP}}=5 \mathrm{~Hz}, \mathrm{ArC}\right), 127.51\left(\mathrm{~d}, J_{\mathrm{CP}}=4 \mathrm{~Hz}\right.$, $\operatorname{ArC}), 128.35$ (s, $\operatorname{ArC}$ ), 130.45 (d, $\left.J_{\mathrm{CP}}=13 \mathrm{~Hz}, \operatorname{ArC}\right), 130.40$ (s, $\operatorname{ArC}$ ), 130.50 (s, $\operatorname{ArC}$ ), 131.12 $\left(\mathrm{dd}, J_{\mathrm{CP}}=21 \mathrm{~Hz}, J_{\mathrm{CP}}=3 \mathrm{~Hz}, \operatorname{Ar} C\right), 131.53\left(\mathrm{~d}, J_{\mathrm{CP}}=3 \mathrm{~Hz}, \operatorname{ArC}\right), 152.26\left(\mathrm{~d}, J_{\mathrm{CP}}=18 \mathrm{~Hz}\right), 162.96$ $\left(\mathrm{dd}, J_{\mathrm{CP}}=8 \mathrm{~Hz}, J_{\mathrm{CP}}=3 \mathrm{~Hz}, \operatorname{ArC}\right), 167.31(\mathrm{~s}, \operatorname{ArC}), 167.59$ (s, $\operatorname{ArC}$ ). Anal. Calcd. for $\mathrm{C}_{28} \mathrm{H}_{41} \mathrm{ClP}_{2} \mathrm{Pd}: \mathrm{C}, 57.84 ; \mathrm{H}, 7.11$. Found: C, $57.55 \mathrm{H}, 7.48$.

\subsection{X-ray single crystal diffraction}

The data were collected on a Bruker APEX-II diffractometer with a monochromated Mo $\mathrm{K} \alpha$ or $\mathrm{Cu} \mathrm{K} \alpha$ radiation. ${ }^{31}$ Data were corrected for absorption and polarized effects and analyzed for space group determination..$^{31,32}$ The structure was solved by direct methods (SHELXS) ${ }^{33}$ and refined by full-matrix least squares techniques against $\mathrm{F}_{0}{ }^{2}$ (SHELXL-97). ${ }^{34}$ Unless noted, all hydrogen atoms were generated in calculated positions. Mercury was used for structure representations. ${ }^{35}$ 


\section{Results and discussion}

The ligand precursor, (Z)-2,3-bis(2-bromophenyl)-2-butene, can be synthesized via the McMurry coupling reaction of 2'-bromoacetophenone. ${ }^{15,30}$ Both the cis and trans isomers are synthesized; they can be separated through the trituration of the crude residue with hexanes. This results in the precipitation of the trans isomer, which can be filtered off leaving behind the pure cis analogue. ${ }^{15,30}$ The final product (1, 2,2' bis(di-iso-propylphosphino)-cis-diphenyl-1,2dimethylethene, $c \mathrm{PCMe}=\mathrm{CMeP})$ is synthesized through the lithium halogen exchange of the ligand precursor, followed by treatment with di-iso-propyl phosphine chloride (Scheme 1). Compound $c \mathrm{PCMe}=\mathrm{CMeP}$ is identified in its ${ }^{31} \mathrm{P} \mathrm{NMR}$ spectrum by a singlet at $-1.86 \mathrm{ppm}$. The high symmetry of the compound is further reflected in its ${ }^{1} \mathrm{H}$ NMR spectrum: the backbone methyl groups resonate equivalently as a singlet at $2.25 \mathrm{ppm}$.

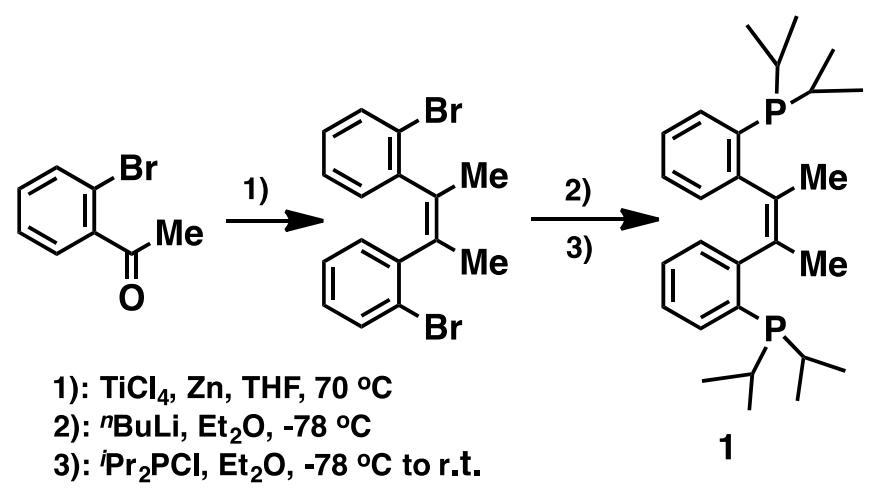

Scheme 1. Synthesis of $c \mathrm{PCMe}=\mathrm{CMeP}$. 
Initially, the synthesis of a palladium( $(0)$ species was carried out. Reacting $c \mathrm{PCMe}=\mathrm{CMeP}$ with one equivalent of $\left[\left\{(\mathrm{TMS}) \mathrm{CH}_{2}\right\}_{2} \mathrm{Pd}(\mathrm{COD})\right]$ in diethyl ether at room temperature rapidly leads to the formation of a three coordinate $\mathrm{Pd}(0)$ species, $[(c \mathrm{PCMe}=\mathrm{CMeP}) \mathrm{Pd}], 2$ (Scheme 2$)$. Characterization of $\mathbf{2}$ by NMR spectroscopy revealed equivalent phosphine environments as a singlet at $48.6 \mathrm{ppm}$ in the ${ }^{31} \mathrm{P}$ NMR spectrum and $C_{s}$ symmetry in the ${ }^{1} \mathrm{H}$ NMR spectrum. The olefin methyl groups resonate as a virtual triplet due to coupling with the phosphines through the metal center at $2.21 \mathrm{ppm}$. The high symmetry in the NMR spectra is indicative of a monomeric instead of a dimeric species. This contrasts the dimeric palladium(0) species that was synthesized previously with $t \mathrm{PCH}=\mathrm{CHP}$, when the olefin and phosphine from one ligand coordinate to the metal center, along with a phosphine from an additional ligand. ${ }^{11}$ The different coordination mode for $c \mathrm{PCMe}=\mathrm{CMeP}$ is attributed to the increased strain of the ligand backbone as well as steric factors. The solution and solid state structures agree, as determined by X-ray crystallography (Figure 1); a severely distorted trigonal planar geometry is observed. The metal center is bound to the olefin, as indicated by the Pd-(C(1)-C(2) centroid) distance of $2.076 \AA$, as well as the elongation of the olefin C-C distance to 1.404(3) $\AA$ compared to typical olefin distances of approximately $1.34 \AA \AA^{36,37}$ The $\mathrm{P}(1)-\mathrm{Pd}-\mathrm{P}(2)$ angle of $140.70(2)^{\circ}$, as well as the sum of angles around the metal center $\left(344.59^{\circ}\right)$, illustrates the degree of distortion from an ideal trigonal planar geometry. Our group has previously synthesized a monomeric trigonal planar palladium( $(0)$ species with the wide bite angle ligand ${ }^{i} \mathrm{Pr}_{2} \mathrm{P}\left(o-\mathrm{C}_{6} \mathrm{H}_{4}-\mathrm{CH}_{2} \mathrm{CH}_{2}-o^{\prime}-\mathrm{C}_{6} \mathrm{H}_{4}\right) \mathrm{P}^{i} \mathrm{Pr}_{2}, \mathrm{~L}^{2}{ }^{38}$ The metal center is bound to an olefin of a dba ligand, as well as to the two phosphine donors on $\mathrm{L}^{2}$. The non-tethered olefin in that situation allows for a more optimal trigonal planar geometry of the metal center $(\mathrm{P}-\mathrm{Pd}-\mathrm{P}=117.117(17)){ }^{38}$ Examples of ligands containing two phosphines and a $\pi$ system donor in the same ligand framework were reported. ${ }^{39,40}$ Agapie and coworkers 
described a tris(phosphine) triphenyl benzene ligand that supports a palladium(0) metal center. Palladium coordinates to two phosphines from the ligand and to the central phenyl ring in an $\eta^{2}$ fashion. Contrasting compound 2, a smaller P-Pd-P angle is observed $\left(128.78^{\circ}\right)$ due to the increased flexibility of their ligand side arms. ${ }^{39}$ In addition, it was reported that 3,5-bis(2phosphinophenyl)-pyridine exhibits less flexibility than the tris(phosphine) triphenyl benzene. ${ }^{39}$ The coordination sphere of palladium(0) when supported by 3,5-bis(2-phosphinophenyl)pyridine consists of the two phosphine donors and an $\eta^{1}-\mathrm{N}$ interaction to the central pyridine ring. Similarly to 2 , the P-Pd-P angle is much wider $\left(155.65^{\circ}\right)$ than $120^{\circ}$ however, the overall geometry is best described as T shaped in that situation. ${ }^{40}$

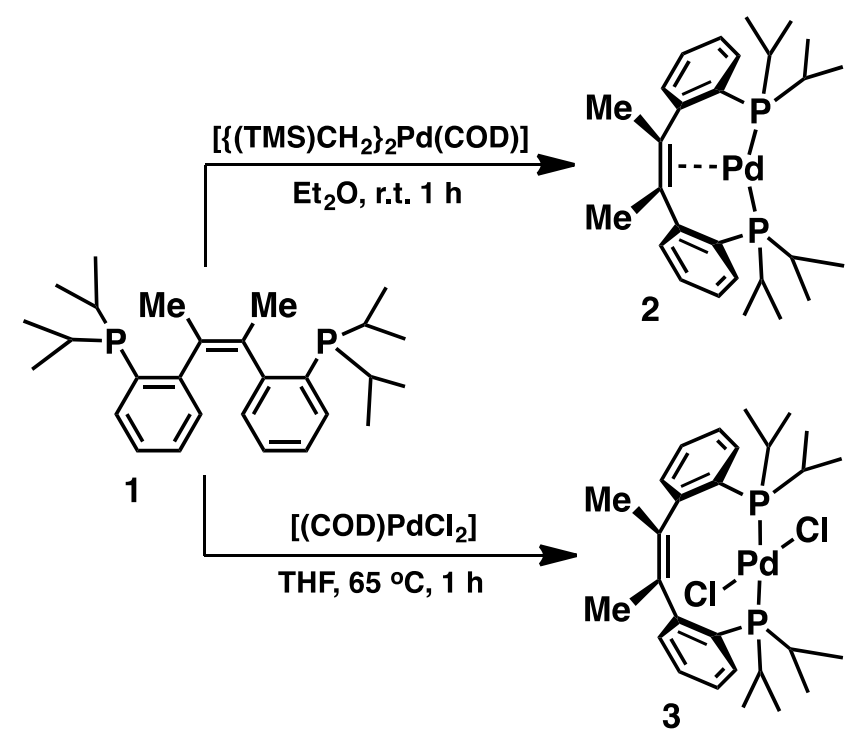

Scheme 2. Synthesis of 2 and 3. 


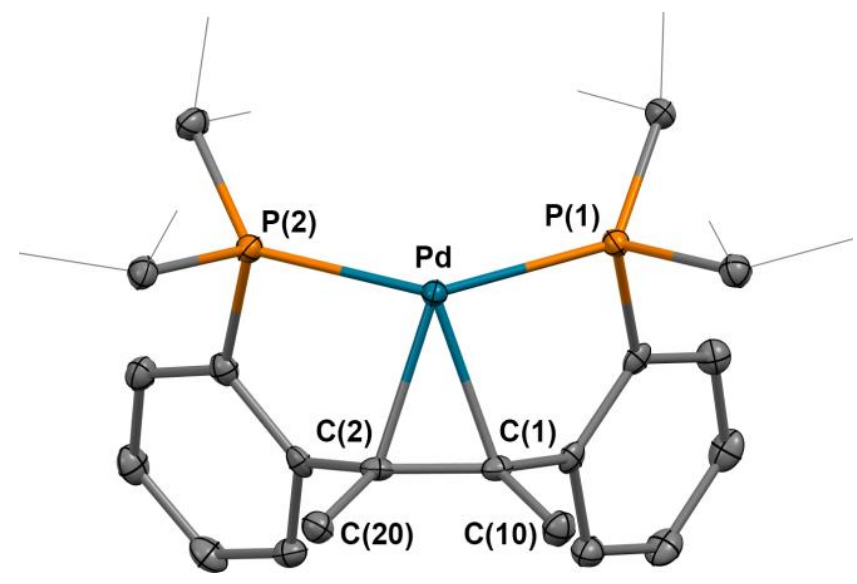

Figure 1. Thermal-ellipsoid (50\% probability level) representation of $[(c \mathrm{PCMe}=\mathrm{CMeP}) \mathrm{Pd}](2)$. Hydrogen atoms and the solvent molecule were omitted for clarity. Selected distances $(\AA)$ and angles $\left({ }^{\circ}\right)$ : $\mathrm{Pd}-\mathrm{P}(1)=2.2748(6), \mathrm{Pd}-\mathrm{P}(2)=2.2892(6), \mathrm{Pd}-\mathrm{C}(1)=2.198(2), \mathrm{Pd}-\mathrm{C}(2)=2.186(2)$ $\mathrm{C}(1)-\mathrm{C}(2)=1.404(3), \mathrm{P}(1)-\mathrm{Pd}-\mathrm{P}(2)=140.70(2), \mathrm{C}(1)-\mathrm{Pd}-\mathrm{C}(2)=37.35(8) \mathrm{C}(1)-\mathrm{Pd}-\mathrm{P}(1)=$ 84.08(6), C(2)-Pd-P(2) = 83.82(6).

Contrasting 2, complexes formed with palladium(II) exhibit a dissociated olefin backbone. Reacting $c \mathrm{PCMe}=\mathrm{CMeP}$ with $\left[(\mathrm{COD}) \mathrm{PdCl}_{2}\right]$ results in a distorted square planar species, $\left[(c \mathrm{PCMe}=\mathrm{CMeP}) \mathrm{PdCl}_{2}\right](3$, Scheme 2$)$. The ${ }^{1} \mathrm{H}$ NMR spectrum reveals a $C_{s}$ symmetry and supports the dissociated state of the olefin through the loss of coupling from the backbone methyl groups to the phosphines. In the ${ }^{31} \mathrm{P}$ NMR spectrum, the phosphines resonate equivalently as a singlet at $68.7 \mathrm{ppm}$. The solid state molecular structure depicts a distorted square planar compound (Figure 2). The $\mathrm{P}(1)-\mathrm{Pd}-\mathrm{P}(2)$ angle $\left(162.75(4)^{\circ}\right)$ is reduced from the optimal $180^{\circ}$ due to the close proximity of the ligand backbone to the metal center $(\mathrm{Pd}-(\mathrm{C}(1)-\mathrm{C}(2)$ centroid $)=$ $3.076 \AA$ A). The coordination mode of the ligand 1 in this palladium(II) complex is different than the one observed for the trans-analogue, $t \mathrm{PCMe}=\mathrm{CMeP}$, which exhibited a coordinated olefin in the resulting cationic complex. ${ }^{11}$ In this case, the strained geometry of the backbone disfavors 
coordination of the olefin, allowing the four-coordinate species to be neutral. Similarly rigid ligands have been prepared by Vogt and coworkers. ${ }^{41}$ The diphosphine 1,2-bis(2(diphenylphosphino)phenyl)benzene is structurally similar to $c \mathrm{PCMe}=\mathrm{CMeP}$, however, the central olefin moiety of the latter is replaced by a central ortho substituted arene group in the former. Similarly to our observations, only a bidentate coordination of 1,2-bis(2(diphenylphosphino)phenyl)benzene to group $10, \mathrm{~d}^{8}$ metal centers was observed. However, cis coordination was observed instead of the trans coordination observed with $c \mathrm{PCMe}=\mathrm{CMeP}^{41}$ Diphosphine ligands that coordinate in a cis arrangement are more numerous than trans coordinating ligands. ${ }^{42,43}$ The excessive flexibility of the larger backbones required for trans coordination can often lead to the formation of dimers and oligomers. Additionally, the activation of the backbone of such large ligands can often lead to tridentate supporting ligands, making bidentate trans coordinating ligands more elusive than their cis coordinating counterparts. ${ }^{42,43}$

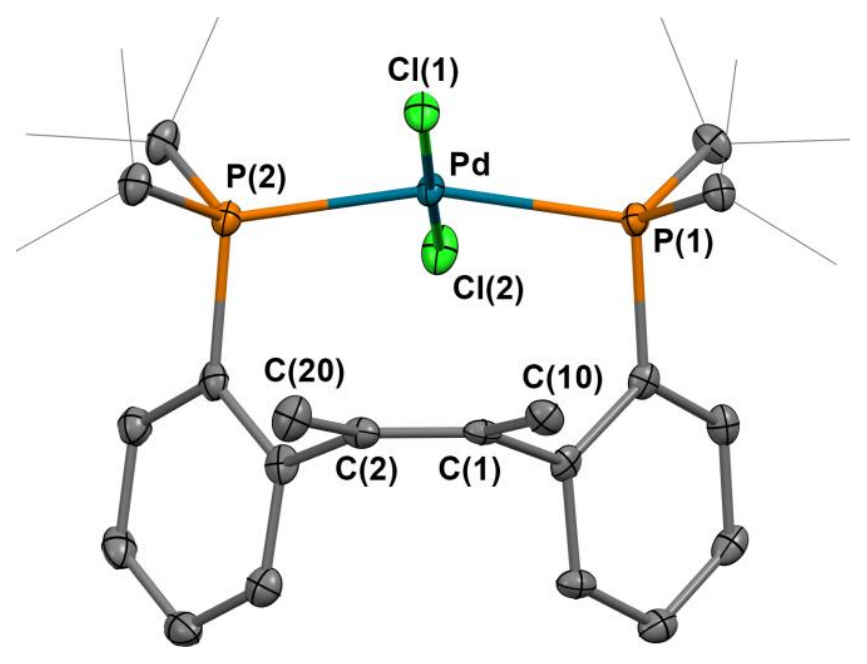


Figure 2. Thermal-ellipsoid (50\% probability level) representation of $\left[(c \mathrm{PCMe}=\mathrm{CMeP}) \mathrm{PdCl}_{2}\right]$ (3). Hydrogen atoms were omitted for clarity. Selected distances $(\AA)$ and angles $\left(^{\circ}\right)$ : $\mathrm{Pd}-\mathrm{P}(1)=$ 2.3031(9), $\mathrm{Pd}-\mathrm{P}(2)=2.3019(10), \mathrm{Pd}-\mathrm{Cl}(1)=2.3251(10), \mathrm{Pd}-\mathrm{Cl}(2)=2.3194(10), \mathrm{C}(1)-\mathrm{C}(2)=$ 1.346(5), $\mathrm{P}(1)-\mathrm{Pd}-\mathrm{P}(2)=162.75(4), \mathrm{Cl}(1)-\mathrm{Pd}-\mathrm{Cl}(2)=175.05(4), \mathrm{Cl}(1)-\mathrm{Pd}-\mathrm{P}(1)=95.09(3)$, $\mathrm{Cl}(2)-\mathrm{Pd}-\mathrm{P}(2)=84.73(4)$.

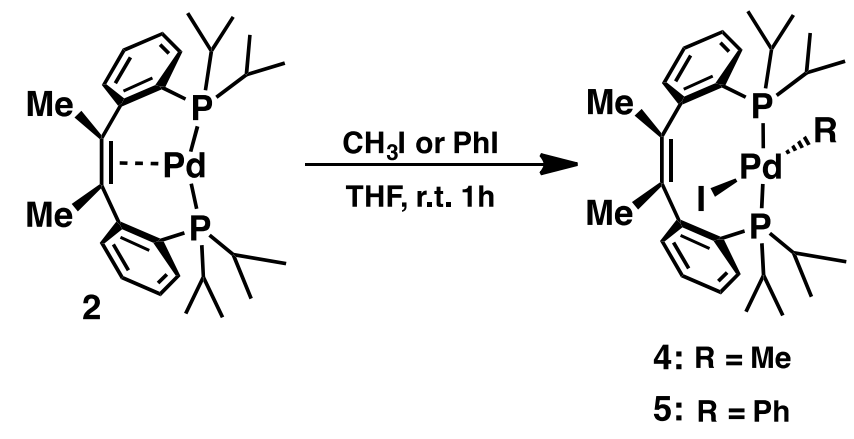

Scheme 3. Oxidative addition of $\mathrm{CH}_{3} \mathrm{I}$ and $\mathrm{PhI}$ to 2.

We observed different coordination modes of the ligand between $\mathbf{2}$ and $\mathbf{3}$. We became interested in investigating oxidative addition reactions of $\mathbf{2}$ in order to determine whether the hemilability of the olefinic backbone could be observed when transitioning from $\operatorname{Pd}(0)$ to $\operatorname{Pd}(\mathrm{II})$ complexes, as well as whether the trans phosphine coordination is preferred over cis in the final $\mathrm{Pd}(\mathrm{II})$ products. These studies were carried out with $\mathrm{CH}_{3} \mathrm{I}, \mathrm{C}_{6} \mathrm{H}_{5} \mathrm{I}, \mathrm{Ph}_{2} \mathrm{SiH}_{2}$, and $\mathrm{HCl}$. In reactions involving of $\mathrm{CH}_{3} \mathrm{I}$ and $\mathrm{C}_{6} \mathrm{H}_{5} \mathrm{I}$, the oxidative addition of the respective substrate results in dissociation of the olefinic backbone generating the square planar compounds $\left[(c \mathrm{PCMe}=\mathrm{CMeP}) \mathrm{PdI}\left(\mathrm{CH}_{3}\right)\right](\mathbf{4})$ and $\left[(c \mathrm{PCMe}=\mathrm{CMeP}) \mathrm{PdI}\left(\mathrm{C}_{6} \mathrm{H}_{5}\right)\right](\mathbf{5}$, Scheme 3$)$. In both species, the backbone methyl groups resonate as a singlet at 2.19 and $2.20 \mathrm{ppm}$ respectively, indicating the dissociated state of the olefin. The solid state molecular structures of both compounds show 
trans phosphine coordination of the ligand as well as a preference for the iodide moiety to add to the metal center syn to the backbone olefin (Figures 3-4). It is likely that this arrangement avoids the close proximity of the backbone that would result if the alkyl (4) or aryl (5) group adds adjacent to the olefin. The presence of the backbone directs the bulkier alkyl and aryl substituent to reside in the anti position to minimize steric interactions. Similarly to compound $\mathbf{3}$, the geometry observed in $\mathbf{4}$ and $\mathbf{5}$ is distorted square planar. The P-Pd-P angles bend to $160.18(4)^{\circ}$ and $157.96(3)^{\circ}$ respectively from the optimal $180^{\circ}$ to avoid steric interactions with the ligand backbone and the additional ligands around the metal center. This bending results in elongated Pd-(C-C centroid) distances of 3.133 and $3.173 \AA$, respectively compared to the distance of $2.076 \AA$ observed upon olefin coordination in $\mathbf{2}$. Interestingly, compound $\mathbf{5}$ has an aryl proton resonating at $4.4 \mathrm{ppm}$ in the ${ }^{1} \mathrm{H}$ NMR spectrum. This surprisingly upfield shift of an aryl proton is justified when examining the solid state molecular structure of the compound (Figure 4). The phenyl group is oriented anti to the olefin, and therefore resides between the two aryl groups in the ligand framework, placing one of the phenyl protons inside the shielding environment of the aromatic rings on the ligand backbone. ${ }^{44}$ It should be noted that the oxidative addition of both substrates results in their trans coordination to the metal center. This is a result of isomerization and was previously observed in complexes with ligands that have a preference for trans coordination. ${ }^{38,45,46}$ A previous report from our group also shows a preference of the diphosphine ${ }^{i} \mathrm{Pr}_{2} \mathrm{P}\left(o-\mathrm{C}_{6} \mathrm{H}_{4}-\mathrm{CH}_{2} \mathrm{CH}_{2}-o^{\prime}-\mathrm{C}_{6} \mathrm{H}_{4}\right) \mathrm{P}^{i} \mathrm{Pr}_{2}\left(\mathrm{~L}^{2}\right)$ for trans coordination to palladium metal centers. In that case, the oxidative addition of $p$-nitrobromobenzene to $\mathrm{L}_{2} \mathrm{Pd}$ resulted in a trans $\mathrm{Pd}(\mathrm{II})$ species as the only observable product. ${ }^{38}$ Additionally, the trans coordinating ligand, dbaphos, reported by Lin and Fairlamb also results in trans-(dbaphos)PdIPh when the oxidative addition of iodobenzene is carried out. ${ }^{45}$ 


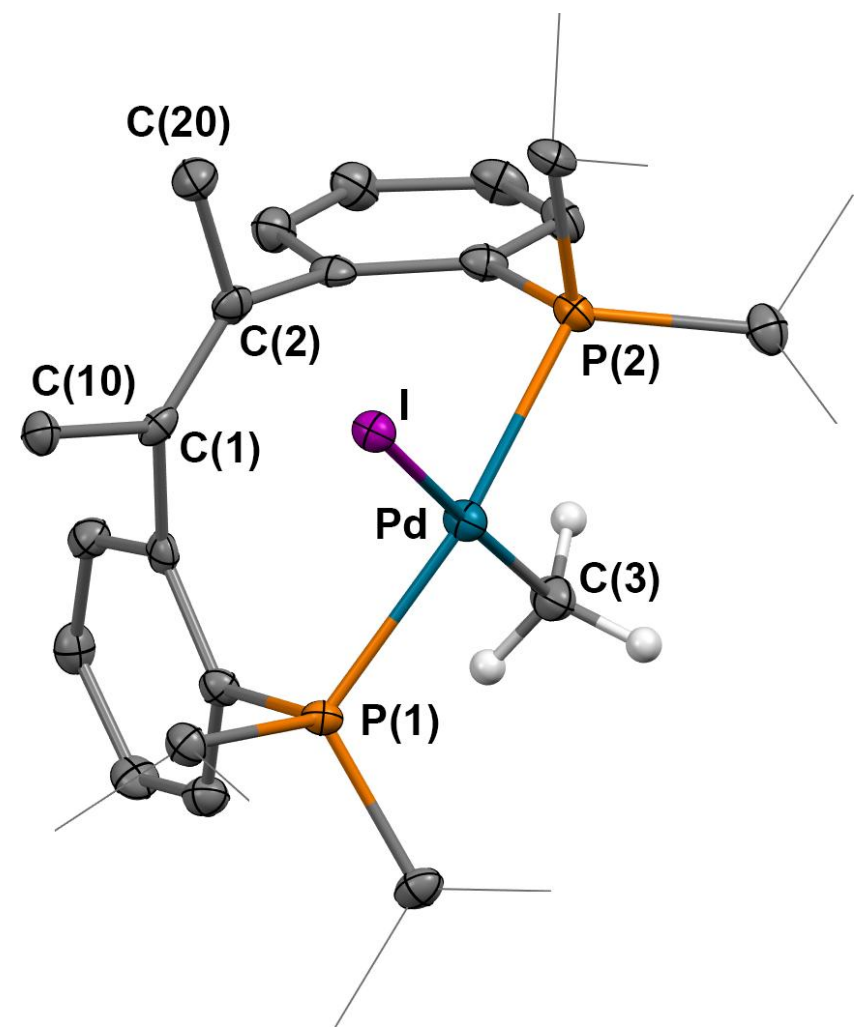

Figure 3. Thermal-ellipsoid (50\% probability level) representation of $\left[(c \mathrm{PCMe}=\mathrm{CMeP}) \mathrm{PdICH}_{3}\right]$ (4). Hydrogen atoms were omitted for clarity. Selected distances $(\AA)$ and angles $\left({ }^{\circ}\right)$ : $\mathrm{Pd}-\mathrm{P}(1)=$ 2.2972(11), $\mathrm{Pd}-\mathrm{P}(2)=2.2944(11), \mathrm{Pd}-\mathrm{C}(3)=2.084(4), \mathrm{Pd}-\mathrm{I}=2.6828(4), \mathrm{C}(1)-\mathrm{C}(2)=1.345(6)$, $\mathrm{P}(1)-\mathrm{Pd}-\mathrm{P}(2)=160.18(4), \mathrm{C}(3)-\mathrm{Pd}-\mathrm{I}=174.47(13), \mathrm{C}(3)-\mathrm{Pd}-\mathrm{P}(1)=84.72(12), \mathrm{I}-\mathrm{Pd}-\mathrm{P}(2)=$ $96.36(3)$. 


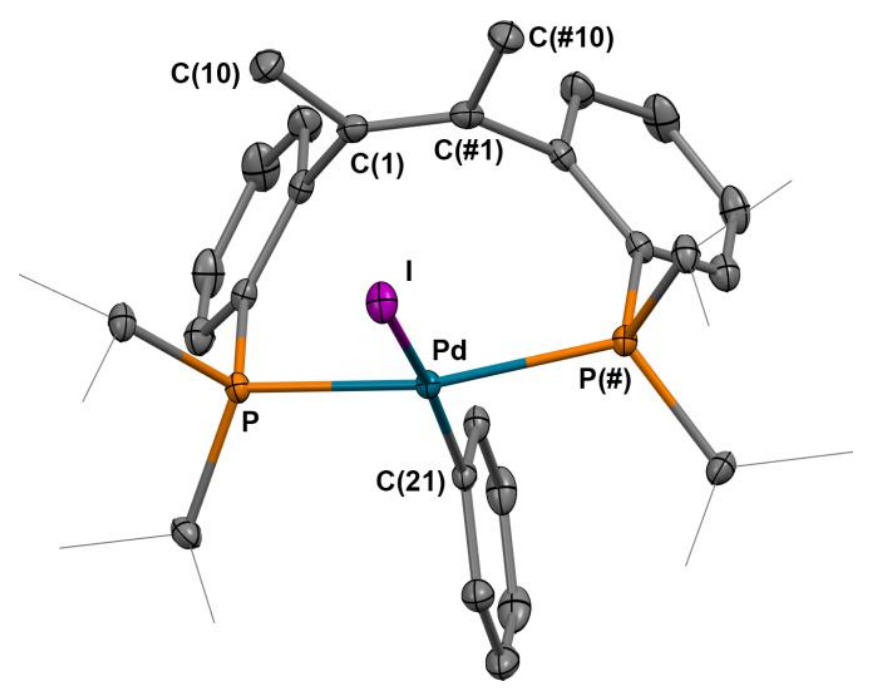

Figure 4. Thermal-ellipsoid $(50 \%$ probability level $)$ representation of $\left[(c \mathrm{PCMe}=\mathrm{CMeP}) \mathrm{PdI}\left(\mathrm{C}_{6} \mathrm{H}_{5}\right)\right](\mathbf{5})$. Hydrogen atoms were omitted for clarity. Selected distances $(\AA)$ and angles $\left(^{\circ}\right)$ : $\mathrm{Pd}-\mathrm{P}=2.3229(5), \mathrm{Pd}-\mathrm{P}(\#)=2.3229(5), \mathrm{Pd}-\mathrm{C}(21)=2.058(3), \mathrm{Pd}-\mathrm{I}=$ 2.6992(3), C(1)-C(\#1) = 1.341(4), P-Pd-P(\#) = 157.96(3) C(21)-Pd-I = 169.85(8), C(21)-Pd-P $=84.981(18), \mathrm{I}-\mathrm{Pd}-\mathrm{P}(\#)=96.666(13)$.

The oxidative addition of $\mathrm{Ph}_{2} \mathrm{SiH}_{2}$ proved to be an interesting case study due to the reversible nature of the Si-H bond activation process. ${ }^{47-49}$ Reacting 2 with $\mathrm{Ph}_{2} \mathrm{SiH}_{2}$ (Scheme 4) results in the rapid formation of a new compound $\left[(c \mathrm{PCMe}=\mathrm{CMeP}) \mathrm{Pd}\left(\mathrm{H}_{2} \mathrm{SiPh}_{2}\right)\right](6)$, which is readily identified in the corresponding ${ }^{31} \mathrm{P}$ NMR spectrum at $37.75 \mathrm{ppm}$ as a singlet. Characterization by ${ }^{1} \mathrm{H}$ NMR spectroscopy indicates a coordinated olefin backbone as attested by the presence of a virtual triplet at $2.16 \mathrm{ppm}$. The $\mathrm{Si}-\mathrm{H}$ protons resonate equivalently and are shifted upfield from free $\mathrm{Ph}_{2} \mathrm{SiH}_{2}(5.02 \mathrm{ppm})$ to $4.55 \mathrm{ppm}$, indicating an interaction with the metal center. Furthermore, single crystal X-ray diffraction showed a distorted square planar metal center resulting from the oxidative addition of a Si-H bond from $\mathrm{Ph}_{2} \mathrm{SiH}_{2}$ (Figure 5). The olefinic backbone is dissociated, as substantiated by the $\mathrm{Pd}-(\mathrm{C}(1)-\mathrm{C}(2)$ centroid) distance of 
$3.130 \AA$, along with the short $\mathrm{C}(1)-\mathrm{C}(2)$ distance of $1.339(3) \AA$. In addition to the contrasting state of the olefin observed in solution and the solid state, another distinction related to the hydrogen atoms originating from the silane was noticed. The solid state structure reveals two distinct environments for these hydrogens atoms, including a palladium hydride and an unchanged Si-H bond which did not undergo a reaction with the metal center. The lack of distinct hydrogen atom signals in the ${ }^{1} \mathrm{H}$ NMR spectrum indicates a fast equilibrium in which the $\mathrm{Si}-\mathrm{H}$ bond cleavage by the metal center is reversible. Similar situations were previously reported for group 10 metals. ${ }^{47-49}$ For example, Hillhouse and coworkers found that reacting the nickel $(0)$ compound (dtbpe)Ni (dtbpe $=$ 1,2-bis(di-tert-butylphosphino)ethane) with dimesitylsilane $\left(\mathrm{Mes}_{2} \mathrm{SiH}_{2}\right)$ led to a species that undergoes a dynamic process involving the reversible oxidative addition of the $\mathrm{Si}-\mathrm{H}$ bond through a $\mathrm{Ni}(0)-\eta^{2}$ silane intermediate. This is observed in the ${ }^{1} \mathrm{H}$ NMR spectrum via the $\mathrm{SiH}$ signal found at $\sim 0 \mathrm{ppm}$, an average of the resonance of the free silane and the nickel hydride resonance ${ }^{47} \mathrm{~A}$ similar observation was reported for a palladium( $(0)$ species by Fink and coworkers. ${ }^{49}$ It was found that the oxidative addition of $\mathrm{R}_{3} \mathrm{SiH}\left(\mathrm{R}_{3}=\mathrm{Ph}_{3}\right.$, $\left.\mathrm{Ph}_{2} \mathrm{Me}, \mathrm{PhMe}_{2}, \mathrm{Et}_{3}\right)$ to the dimeric palladium $(0)$ species $[(\mu \text {-dcpe }) \mathrm{Pd}]_{2}$, $(\mathrm{dcpe}=1,2$,-bis(dicyclohexylphosphino)ethane) produced the dynamic compound $\mathrm{PdH}\left(\mathrm{SiHR}_{3}\right)(\mathrm{dcpe})$. Similarly to the report by Hillhouse and coworkers, the $\mathrm{Si} H$ resonance was found as an average of that for the free silane and a metal hydride species ( -1 to $-2 \mathrm{ppm})$. Comparing their results to the data obtained for complex $\mathbf{6}$, it is concluded that a similar dynamic process is occurring, however, the presence of the olefin backbone heavily pushes the equilibrium toward the $\operatorname{Pd}(0)$ complex. The appearance of the $\mathrm{Si} H$ protons downfield at $4.55 \mathrm{ppm}$ indicates that the weighted average of the species present in solution favors the unreacted $(c \mathrm{PCMe}=\mathrm{CMeP}) \mathrm{Pd}$ or a $\sigma$-bound $\mathrm{Si}-\mathrm{H}$ species. It 
is also of interest to note that $\mathbf{6}$ represents the only case observed so far in which oxidative addition results in cis-coordination of the ligand $\mathbf{1}$.
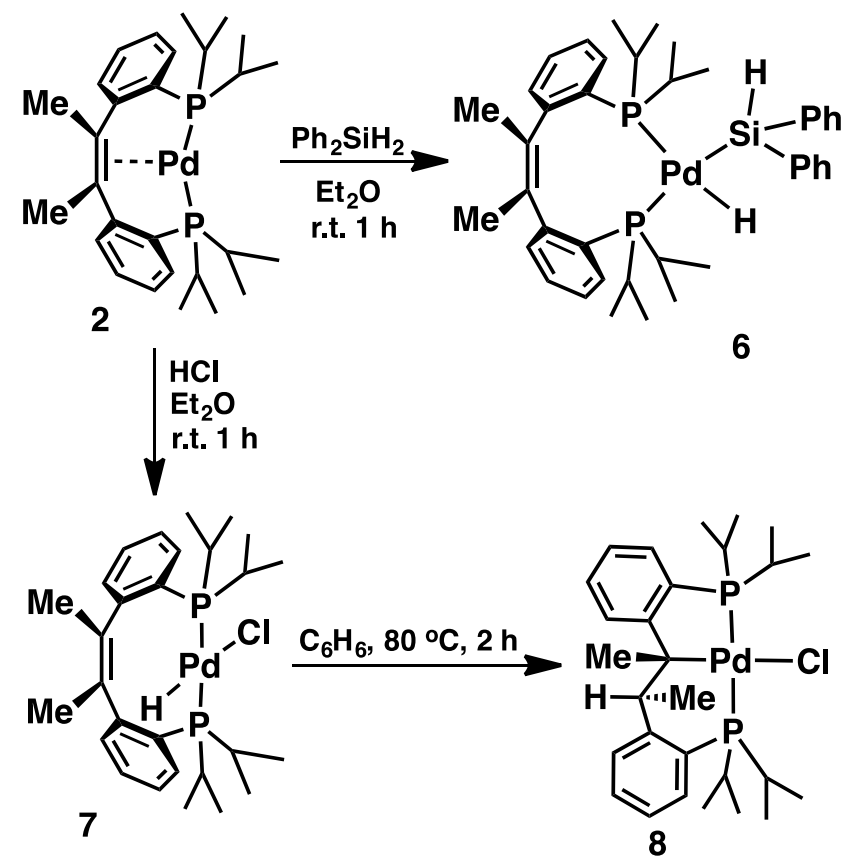

Scheme 4. Syntheses of 6,7 , and 8 . 


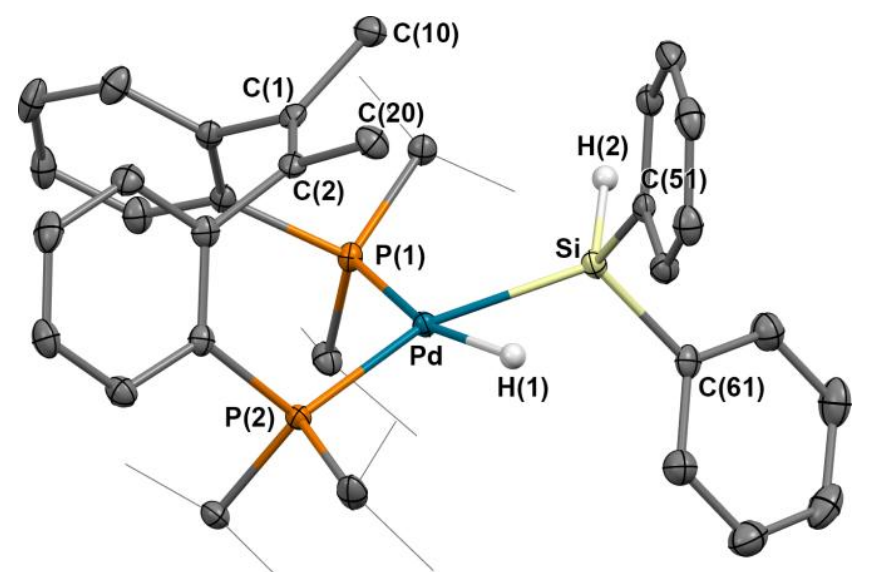

Figure 5. Thermal-ellipsoid $(50 \%$ probability level $)$ representation of $\left[(c \mathrm{PCMe}=\mathrm{CMeP}) \mathrm{PdH}_{2} \mathrm{SiPh}_{2}\right](6)$. Most hydrogen atoms were omitted for clarity. Selected distances $(\AA)$ and angles $\left(^{\circ}\right)$ : Pd-P(1) = 2.3365(6), Pd-P(2) = 2.3759(6), Pd-Si $=2.3298(6), \mathrm{Pd}-$ $\mathrm{H}(1)=1.61(3), \mathrm{Si}-\mathrm{H}(2)=1.41(2), \mathrm{Si}-\mathrm{H}(1)=1.95(3), \mathrm{C}(1)-\mathrm{C}(2)=1.339(3), \mathrm{P}(1)-\mathrm{Pd}-\mathrm{P}(2)=$ 109.955(19), Si-Pd-H(1) = 56.0(9), Si-Pd-P(1) = 103.33(2), H(1)-Pd-P(2) = 91.0(9).

Reacting $[(c \mathrm{PCMe}=\mathrm{CMeP}) \mathrm{Pd}], 2$, with one equivalent of $\mathrm{HCl}$ in diethyl ether at room temperature led to the clean formation of a chloro-hydride species, $[(c \mathrm{PCMe}=\mathrm{CMeP}) \mathrm{PdHCl}](\mathbf{7}$, Scheme 4). The hydride is found upfield in the corresponding ${ }^{1} \mathrm{H}$ NMR spectrum at $-14.58 \mathrm{ppm}$ as a singlet. Coupling between the hydride and the phosphorous nuclei was not observed even at low temperatures. The methyl groups of the ligand backbone resonate as a singlet at $2.34 \mathrm{ppm}$. The lack of coupling between the methyl groups and the phosphines is suggestive of a dissociated olefin moiety. The ${ }^{31} \mathrm{P}$ NMR spectrum displays the equivalent phosphines as a singlet at $37.27 \mathrm{ppm}$. The lack of coupling observed between the hydride and the phosphines, as well as the similar chemical shift observed in the ${ }^{31} \mathrm{P}$ NMR spectrum compared to the other trans 
compounds discussed previously (4-5), indicates that $c \mathrm{PCMe}=\mathrm{CMeP}$ coordinates in a trans fashion.

Compound 7 transformed, after 12 hours, into a new asymmetric compound (8) as indicated by the ${ }^{1} \mathrm{H}$ and ${ }^{31} \mathrm{P}$ NMR spectra. After 4 days at room temperature, the original chlorohydride species, 7, was completely consumed, leading to a clean solution of compound $\mathbf{8}$. This process can also be accomplished by heating the solution to $80{ }^{\circ} \mathrm{C}$ for $2 \mathrm{~h}$ (Scheme 4). The ${ }^{1} \mathrm{H}$ NMR of $\mathbf{8}$ contains five separate methine resonances, as well as two separate peaks for the backbone methyl groups at 1.59 and $1.16 \mathrm{ppm}$, respectively, indicating protonation of the ligand backbone. This conclusion is further supported by the loss of the hydride signal. The inequivalent phosphine environments are found in the ${ }^{31} \mathrm{P}$ NMR spectrum as trans coupling doublets at 66.09 and $18.48 \mathrm{ppm}$, respectively $\left({ }^{2} J_{\mathrm{PP}}=356 \mathrm{~Hz}\right)$. The solid state molecular structure, obtained from a concentrated toluene solution layered with $n$-pentane, confirms the protonation of the ligand backbone with an elongated $\mathrm{C}(1)-\mathrm{C}(2)$ distance of $1.460(7) \AA$, along with an $\mathrm{sp}^{3}$ hybridization of $\mathrm{C}(1)$ and $\mathrm{C}(2)$ (Figure 6). Palladium hydrides are known to be short lived in the presence of an olefin due to the alkene insertion into the $\mathrm{Pd}-\mathrm{H}$ bond. ${ }^{50,51}$ Assessing the stereochemistry of the protonated backbone reveals a syn-addition to the olefin, allowing an intramolecular migratory insertion to be a plausible mechanism for this process. ${ }^{52}$ However, an unlikely intermolecular protonation pathway followed by the isomerization of the backbone could not be ruled out. 


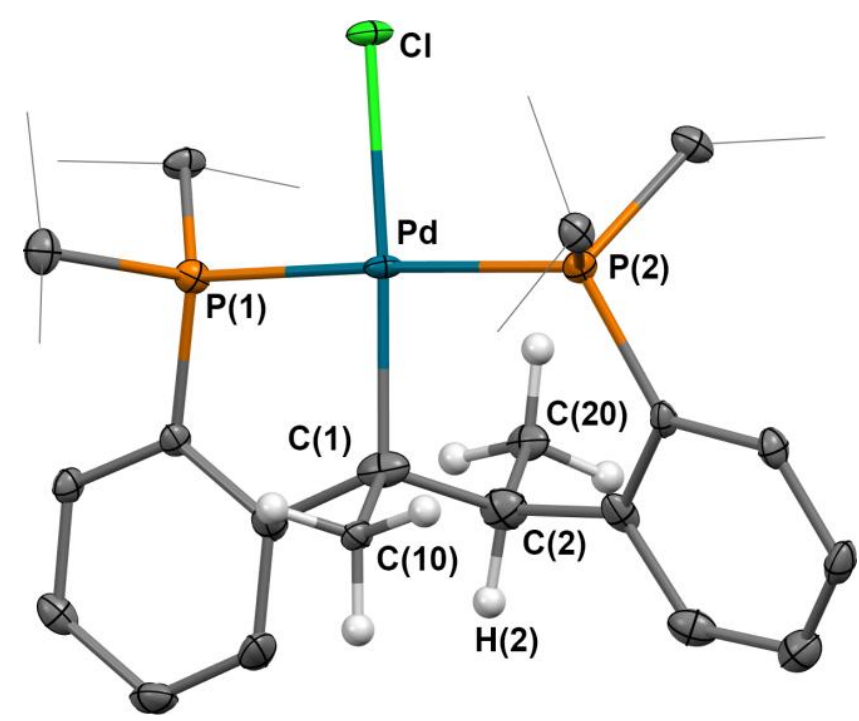

Figure 6. Thermal-ellipsoid (50\% probability level) representation of [(PCMe-CHMeP) $\mathrm{PdCl}]$ (8). Most hydrogen atoms were omitted for clarity. Selected distances $(\AA)$ and angles $\left(^{\circ}\right)$ : $\operatorname{Pd}-$ $\mathrm{P}(1)=2.2704(12), \mathrm{Pd}-\mathrm{P}(2)=2.3140(12), \mathrm{Pd}-\mathrm{Cl}=2.4174(11), \mathrm{Pd}-\mathrm{C}(1) 2.108(5), \mathrm{C}(1)-\mathrm{C}(2)=$ 1.460(7), $\mathrm{P}(1)-\mathrm{Pd}-\mathrm{P}(2)=177.35(5), \mathrm{Cl}-\mathrm{Pd}-\mathrm{C}(1)=174.56(15), \mathrm{Cl}-\mathrm{Pd}-\mathrm{P}(1)=91.08(4), \mathrm{C}(1)-$ $\mathrm{Pd}-\mathrm{P}(2)=93.46(16), \mathrm{Pd}-\mathrm{C}(1)-\mathrm{C}(2)=109.6(4)$.

The formation of $\mathbf{8}$ from $\mathbf{7}$ demonstrates the hydrogen acceptor capabilities of the ligand backbone. Therefore, we decided to study if the ligand could also act as a hydrogen atom donor. Attempts at deprotonating the olefin insertion product to reform the $\operatorname{Pd}(0)$ species (2) by reacting $\mathbf{8}$ with a base failed, likely because of the presence of multiple deprotonation sites. Consequently, the dehydrohalogenation of $\left[(c \mathrm{PCMe}=\mathrm{CMeP}) \mathrm{PdCl}_{2}\right](\mathbf{3})$ was targeted instead. Reacting 3 with one equivalent of benzyl potassium led to the formation of a new asymmetric species after 1 hour (Scheme 5). Characterization of the product, [(PCMe-C $\left.\left.\left(=\mathrm{CH}_{2}\right) \mathrm{P}\right) \mathrm{PdCl}\right](9)$, revealed the deprotonation of a methyl group on the olefin, resulting in the rearrangement of the ligand backbone. The new olefinic protons can be observed in the ${ }^{1} \mathrm{H}$ NMR spectrum as a doublet of 
doublets at 4.85 and $4.63 \mathrm{ppm}$, respectively, due to the coupling with the two non-equivalent phosphines. The ${ }^{31} \mathrm{P}\left\{{ }^{1} \mathrm{H}\right\}$ NMR spectrum shows the two phosphines as trans coupling doublets $(356 \mathrm{~Hz})$ at 68.09 and $18.49 \mathrm{ppm}$, respectively. Crystals suitable for single crystal X-ray diffraction were obtained from a concentrated $n$-pentane solution. The solid state molecular structure (Figure 7) confirms the rearrangement of the backbone upon the deprotonation of a methyl group. The C(2)-C(4) distance of 1.366(5) $\AA$ is indicative of a C-C double bond, while the $\mathrm{C}(1)-\mathrm{C}(2)$ distance of $1.473(5) \AA$ is characteristic of a C-C single bond. The deprotonation of the backbone was found to be reversible. Treating a THF solution of 9 with one equivalent of $\mathrm{HCl}$ cleanly forms 3 .

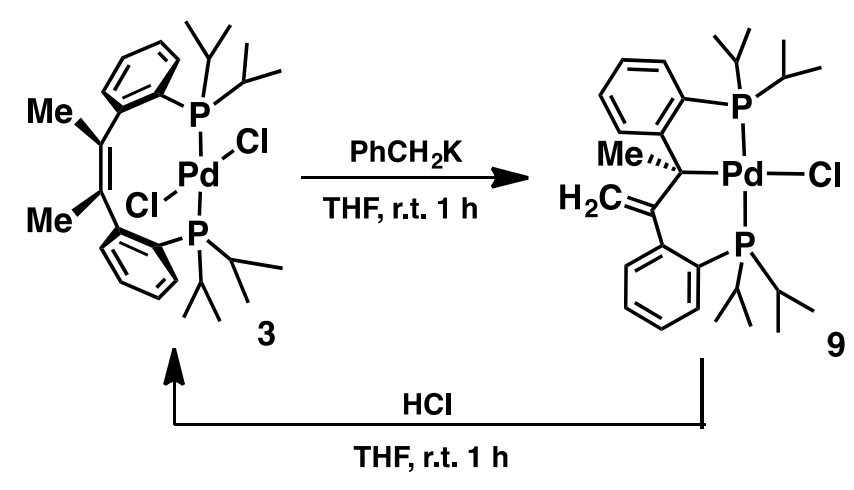

Scheme 5. Synthesis of 9. 


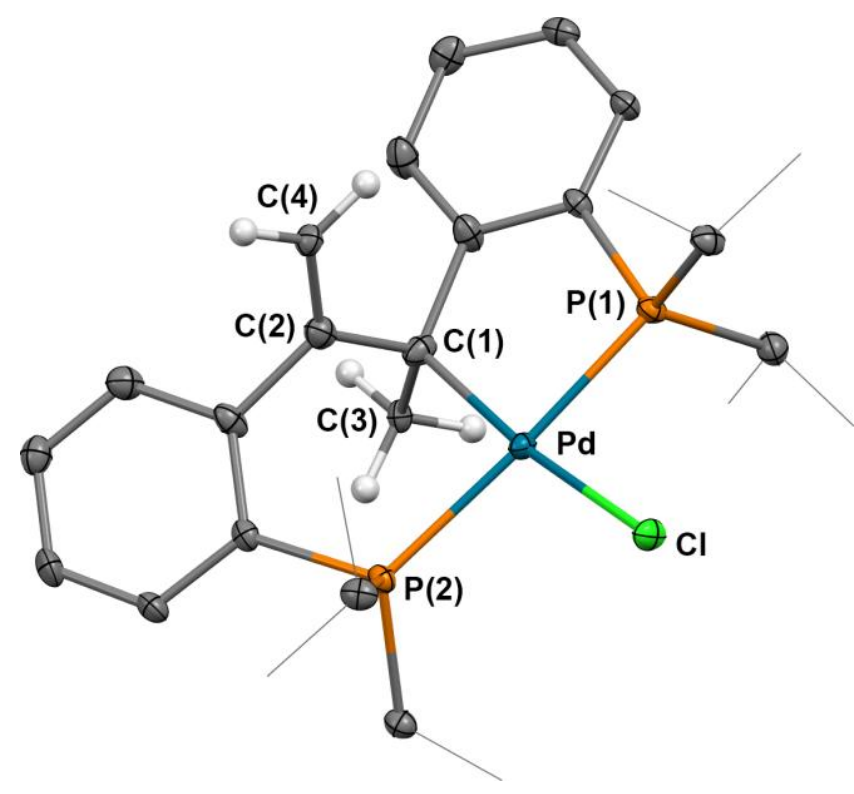

Figure 7. Thermal-ellipsoid $(50 \%$ probability level $)$ representation of $\left[\left(\mathrm{PC}\left(\mathrm{CH}_{3}\right)\right.\right.$ $\left.\left.\mathrm{C}\left(=\mathrm{CH}_{2}\right) \mathrm{P}\right) \mathrm{PdCl}\right](\mathbf{9})$. Most hydrogen atoms were omitted for clarity. Selected distances $(\AA)$ and angles $\left({ }^{\circ}\right): \mathrm{Pd}-\mathrm{P}(1)=2.2815(8), \mathrm{Pd}-\mathrm{P}(2)=2.3434(8), \mathrm{Pd}-\mathrm{Cl}=2.4176(8), \mathrm{Pd}-\mathrm{C}(1)=2.104(3)$, $\mathrm{C}(1)-\mathrm{C}(2)=1.473(5), \mathrm{C}(2)-\mathrm{C}(4)=1.366(5), \mathrm{C}(1)-\mathrm{C}(3)=1.520(5), \mathrm{P}(1)-\mathrm{Pd}-\mathrm{P}(2)=176.62(3)$, $\mathrm{Cl}-\mathrm{Pd}-\mathrm{C}(1)=173.23(10), \mathrm{Cl}-\mathrm{Pd}-\mathrm{P}(1)=91.88(3), \mathrm{C}(1)-\mathrm{Pd}-\mathrm{P}(2)=93.90(11), \mathrm{Pd}-\mathrm{C}(1)-\mathrm{C}(2)=$ 100.6(2), C(1)-C(2)-C(4)=122.7(3).

\section{Conclusions}

The chelating ligand $c \mathrm{PCMe}=\mathrm{CMeP}$ was synthesized in order to study the coordination of the olefinic backbone in several palladium complexes. A hemilabile behavior was observed during the oxidative addition of $\mathrm{CH}_{3} \mathrm{I}, \mathrm{C}_{6} \mathrm{H}_{5} \mathrm{I}, \mathrm{Ph}_{2} \mathrm{SiH}_{2}$, and $\mathrm{HCl}$ to $[(c \mathrm{PCMe}=\mathrm{CMeP}) \mathrm{Pd}]$. Full dissociation of the olefin occurred in all cases except for the silane substrate, when a reversible $\mathrm{Si}-\mathrm{H}$ activation in conjunction with a reversible olefin dissociation were observed. The capabilities of the olefinic moiety of the ligand to act as an $\mathrm{H}$-atom reservoir were probed through its protonation by a $\mathrm{Pd}-\mathrm{H}$ species, as well as in the dehydrohalogenation of 
$\left[(c \mathrm{PCMe}=\mathrm{CMeP}) \mathrm{PdCl}_{2}\right]$ upon the addition of a base. The variety of cooperative behavior observed is promising and applications toward catalysis will be the focus of our studies going forward.

\section{Acknowledgments}

We would like to thank Dr. Allen Oliver for assistance with the crystallography studies. This work was supported by the National Science Foundation (CHE-1552397) and by the University of Notre Dame.

\section{Appendix A. Supplementary material}

CCDC 1487886-1487892 contain the supplementary crystallographic data for $\mathbf{2 - 6 , 8}$, and $\mathbf{9}$. These data can be obtained free of charge from The Cambridge Crystallographic Data Centre via www.ccdc.cam.ac.uk/data_ request/cif. Supplementary data associated with this article can be found in the online version at http://dx.doi.org/.

\section{References}

(1) Annibale, V. T.; Song, D. R. Soc. Chem. Adv. 2013, 3, 11432.

(2) van der Vlugt, J. I. Eur. J. Inorg. Chem. 2012, 363.

(3) Grutzmacher, H. Angew. Chem. Int. Ed. 2008, 47, 1814.

(4) Devillard, M.; Bouhadir, G.; Bourissou, D. Angew. Chem. Int. Ed. 2015, 54, 730.

(5) Musa, S.; Shaposhnikov, I.; Cohen, S.; Gelman, D. Angew. Chem. Int. Ed. 2011, $50,3533$.

(6) Rodríguez-Lugo, R. E.; Trincado, M.; Vogt, M.; Tewes, F.; Santiso-Quinones, G.; Grützmacher, H. Nat. Chem. 2013, 5, 342.

(7) Gunanathan, C.; Ben-David, Y.; Milstein, D. Science 2007, 317, 790.

(8) Zhang, J.; Leitus, G.; Ben-David, Y.; Milstein, D. J. Am. Chem. Soc. 2005, 127, 10840.

(9) Gunanathan, C.; Milstein, D. Acc. Chem. Res. 2011, 44, 588.

(10) Lin, S. B.; Day, M. W.; Agapie, T. J. Am. Chem. Soc. 2011, 133, 3828.

(11) Barrett, B. J.; Iluc, V. M. Organometallics 2014, 33, 2565. 
(12) Bennett, M. A.; Clark, P. W.; Robertson, G. B.; Whimp, P. O. J. Chem. Soc., Chem. Commun. 1972, 1011.

(13) Bennett, M. A.; Corlett, S.; Robertson, G. B.; Steffen, W. L. Aust. J. Chem. 1980, 205.

(14) Bennett, M. A.; Johnson, R. N.; Tomkins, I. B. J. Organomet. Chem. 1976, 118,

(15) Barrett, B. J.; Iluc, V. M. Inorg. Chem. 2014, 53, 7248.

(16) Comanescu, C. C.; Iluc, V. M. Organometallics 2015, 34, 4684.

(17) Comanescu, C. C.; Iluc, V. M. Chem. Commun. 2016, DOI: 10.1039/C5CC09468B.

(18) Cui, P.; Comanescu, C. C.; Iluc, V. M. Chem. Commun. 2015, 51, 6206.

(19) Comanescu, C. C.; Iluc, V. M. Organometallics 2014, 33, 6059.

(20) Peng, C.; Iluc, V. M. Chem. Sci. 2015, 6, 7343.

(21) Comanescu, C. C.; Vyushkova, M.; Iluc, V. Chem. Sci. 2015, 6, 4570.

(22) Braunstein, P.; Naud, F. Angew. Chem. Int. Ed. 2001, 40, 680.

(23) Zeng, M.; Li, L.; Herzon, S. B. J. Am. Chem. Soc. 2014, 136, 7058.

(24) Anderson, J. S.; Rittle, J.; Peters, J. C. Nature 2013, 501, 84.

(25) Fulmer, G. R.; Kaminsky, W.; Kemp, R. A.; Goldberg, K. I. Organometallics 2011, 30, 1627. 2516.

(26) Deckers, P. J. W.; Hessen, B.; Teuben, J. H. Angew. Chem. Int. Ed. 2001, 40,

(27) van der Vlugt, J. I.; Pidko, E. A.; Vogt, D.; Lutz, M.; Spek, A. L.; Meetsma, A. Inorg. Chem. 2008, 47, 4442.

(28) Moxham, G. L.; Randell-Sly, H. E.; Brayshaw, S. K.; Woodward, R. L.; Weller, A. S.; Willis, M. C. Angew. Chem. 2006, 118, 7780.

(29) Lee, H. G.; Milner, P. J.; Buchwald, S. L. Org. Lett. 2013, 15, 5602.

(30) Yamazaki, S.; Yoshimura, T.; Yamabe, S.; Arai, T.; Tamura, H. J. Org. Chem. 1990, 55, 263.

(31) Bruker AXS (2014). APEX-2. bruker-Nonius AXS, Madison, Wisconsin, USA.

(32) Krause, L.; Herbst-Irmer, R.; Sheldrick, G. M.; Stalke, D. J. Appl. Cryst. 2015, 48, 3.

(33) Sheldrick, G. Acta Cryst. 2015, A71, 3.

(34) Sheldrick, G. Acta Cryst. 2015, C71, 3.

(35) Macrae, C. F.; Bruno, I. J.; Chisholm, J. A.; Edgington, P. R.; McCabe, P.; Pidcock, E.; Rodriguez-Monge, L.; Taylor, R.; van de Streek, J.; Wood, P. A. J. Appl. Cryst. 2008, 41, 466.

(36) Canovese, L.; Visentin, F.; Chessa, G.; Uguagliati, P.; Dolmella, A. J. Organomet. Chem. 2000, 601, 1.

(37) van Asselt, R.; Elsevier, C. J.; Smeets, W. J. J.; Spek, A. L. Inorg. Chem. 1994, 33, 1521.

(38) Comanescu, C. C.; Iluc, V. M. Inorg. Chem. 2014, 53, 8517.

(39) Suseno, S.; Agapie, T. Organometallics 2013, 32, 3161.

(40) Horak, K. T.; VanderVelde, D. G.; Agapie, T. Organometallics 2015, 34, 4753.

(41) van der Vlugt, J. I.; van Duren, R.; Batema, G. D.; den Heeten, R.; Meetsma, A.; Fraanje, J.; Goubitz, K.; Kamer, P. C. J.; van Leeuwen, P. W. N. M.; Vogt, D. Organometallics 2005, 24, 5377. 
(42) Bessel, C. A.; Aggarwal, P.; Marschilok, A. C.; Takeuchi, K. J. Chem. Rev. 2001, $101,1031$.

(43) Freixa, Z.; van Leeuwen, P. W. N. M. Coord. Chem. Rev. 2008, 252, 1755.

(44) Boekelheide, V.; Hylton, T. A. J. Am. Chem. Soc. 1970, 92, 3669.

(45) Jarvis, A. G.; Sehnal, P. E.; Bajwa, S. E.; Whitwood, A. C.; Zhang, X.; Cheung, M. S.; Lin, Z.; Fairlamb, I. J. S. Chem. Eur. J. 2013, 19, 6034.

(46) Yin, J.; Buchwald, S. L. J. Am. Chem. Soc. 2002, 124, 6043.

(47) Iluc, V. M.; Hillhouse, G. L. Tetrahedron 2006, 62, 7577.

(48) Nakata, N.; Fukazawa, S.; Kato, N.; Ishii, A. Organometallics 2011, 30, 4490.

(49) Boyle, R. C.; Mague, J. T.; Fink, M. J. J. Am. Chem. Soc. 2003, 125, 3228.

(50) Takaya, J.; Iwasawa, N. J. Am. Chem. Soc. 2008, 130, 15254.

(51) Takaya, J.; Kirai, N.; Iwasawa, N. J. Am. Chem. Soc. 2011, 133, 12980.

(52) Doherty, N. M.; Bercaw, J. E. J. Am. Chem. Soc. 1985, 107, 2670. 

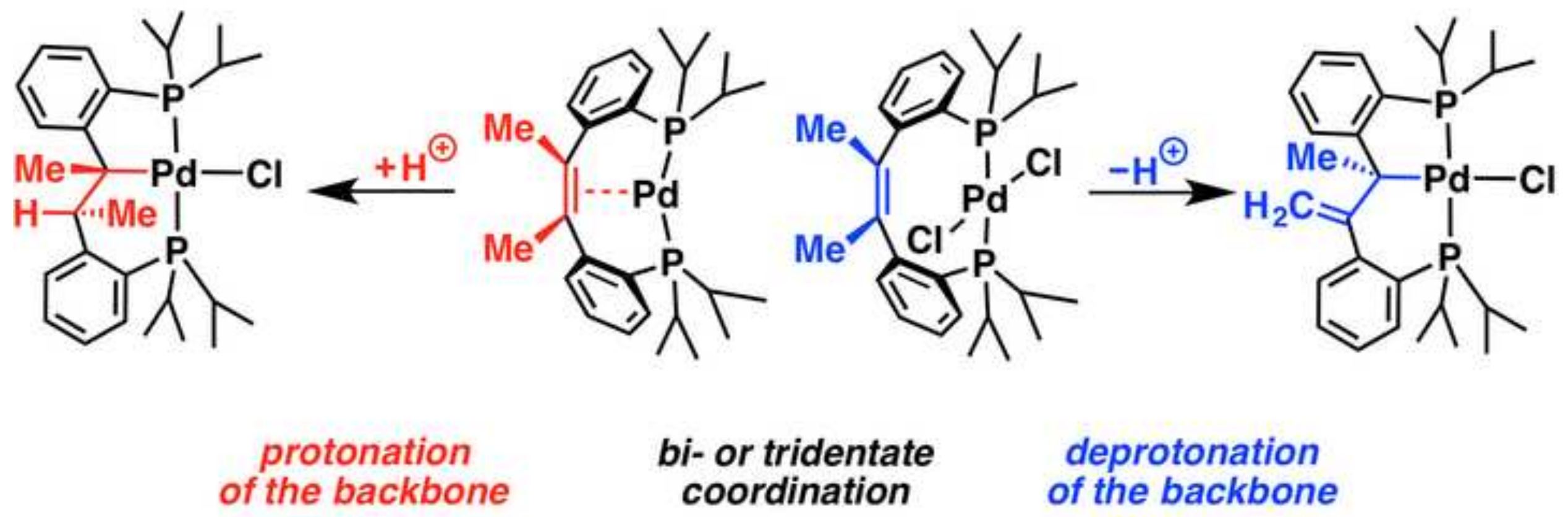

The hemilability and the reactivity of the olefinic backbone of a chelate ligand was studied in several palladium complexes. 